\title{
Solution Structures and Molecular Associations of a Peptide-Based Catalyst for the Stereoselective Baeyer- Villiger Oxidation
}

\author{
Nadia C. Abascal and Scott J. Miller* \\ Department of Chemistry, Yale University, New Haven, CT 06520-8107, United States \\ *E-mail: scott.miller@yale.edu
}

Supporting Information

Table of Contents

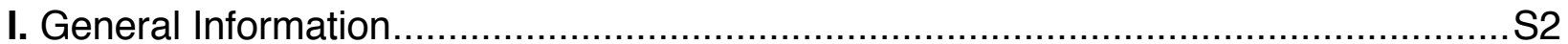

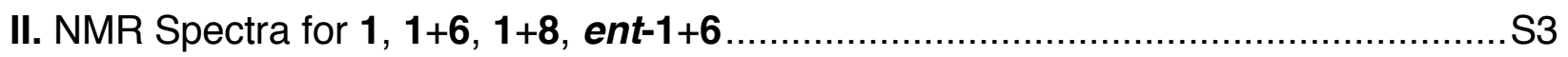

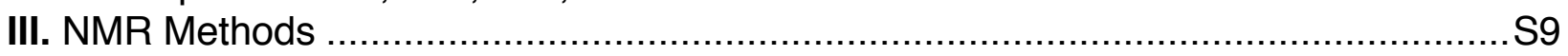

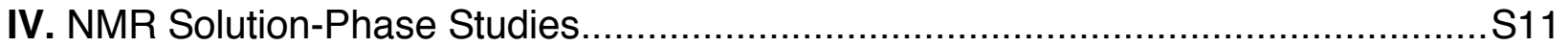

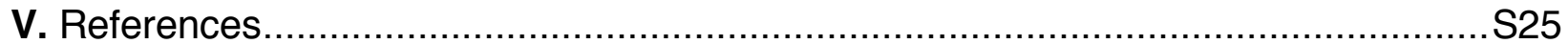




\section{General Information}

All NMR spectra were collected in deuterated chloroform $\left(\mathrm{CDCl}_{3}\right)$, purchased from Cambridge Isotope Laboratories. NMR data ( $\left.{ }^{1} \mathrm{H}, \mathrm{COSY}, \mathrm{ROESY}\right)$ was collected on $400 / 600 \mathrm{MHz}$ instruments at ambient temperature.

Room temperature (rt) is defined as $21-23{ }^{\circ} \mathrm{C}$. All reagents were purchased from commercial suppliers and used without further purification, unless otherwise noted. Routine ${ }^{1} \mathrm{H}$-NMR spectra were recorded on Agilent $500 \mathrm{MHz}$ spectrometers at ambient temperature. Spectra were processed with MestReNova 10.0.2 using the automatic phasing and Bernstein third order polynomial baseline correction capabilities. Splitting was determined using the automatic multiplet analysis function with intervention as necessary. Spectral data are reported as follows: chemical shift (multiplicity [singlet (s), doublet $(d)$, triplet $(t)$, quartet $(q)$, pentet $(p)$, multiplet $(m)$, doublet of doublets $(d d)$, doublet of doublet of doublets (ddd), doublet of triplet of doublets (dtd), doublet of triplets (dt), triplet of doublets (td), etc.], coupling constant, integration). Chemical shifts are reported in ppm $(\delta)$, and coupling constants are reported in $\mathrm{Hz}$. ${ }^{1} \mathrm{H}$-Resonances are referenced to solvent residual peaks for $\mathrm{CDCl}_{3}(7.26 \mathrm{ppm}){ }^{1}$ Infrared spectra were recorded on a Nicolet $6700 \mathrm{FT}-\mathrm{IR}, v_{\max }\left(\mathrm{cm}^{-1}\right)$ and are partially reported in accordance with convention. Analytical thin-layer chromatography (TLC) was performed using EMD Millipore silica gel 60 F254 precoated plates $(0.25 \mathrm{~mm}$ thickness) and were visualized by irradiation with UV light $(254 \mathrm{~nm})$ or staining with $\mathrm{KMnO}_{4}$. TLC $\mathrm{R}_{f}$ values are reported. Reversed phase chromatography used C-18 silica and was performed on a Biotage Isolera One purification system. The gradient of the eluent $(\nabla)$ is given as \% strong solvent/column volume (CV). Optical rotation was recorded on a Perkin Elmer Polarimeter 341 at the D line (1.0 dm path length). Normal phase HPLC was performed on an Agilent 1100 series chromatograph equipped with a photodiode array detector $(210 \mathrm{~nm}, 230 \mathrm{~nm}$, and $254 \mathrm{~nm})$. Peptide sequence determination was performed with UPLC-MS on a Waters Acquity UPLC $®$ BEH C8 $(1.7 \mu \mathrm{m}, 2.1 \times 100 \mathrm{~mm})$ column on a Waters XEVO instrument equipped with ESI, a QToF mass spectrometer, and a photodiode array detector. Low resolution mass spectrometry (MS) was also acquired by this method. High resolution mass spectrometry (HRMS) used electrospray ionization (ESI) and was conducted by the Mass Spectrometry Laboratory at the University of Illinois at Urbana-Champaign.

Note: The characterization for all compounds reported below has already been reported. ${ }^{2}$ 
II. NMR Spectra for 1, 1+6, 1+8, ent-1+6

Peptide 1

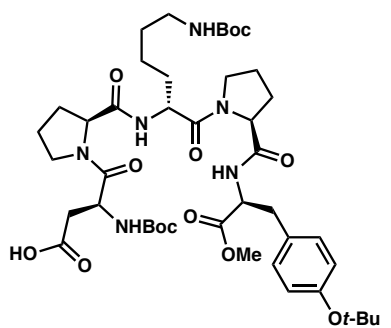

${ }^{1} \mathrm{H}$ NMR

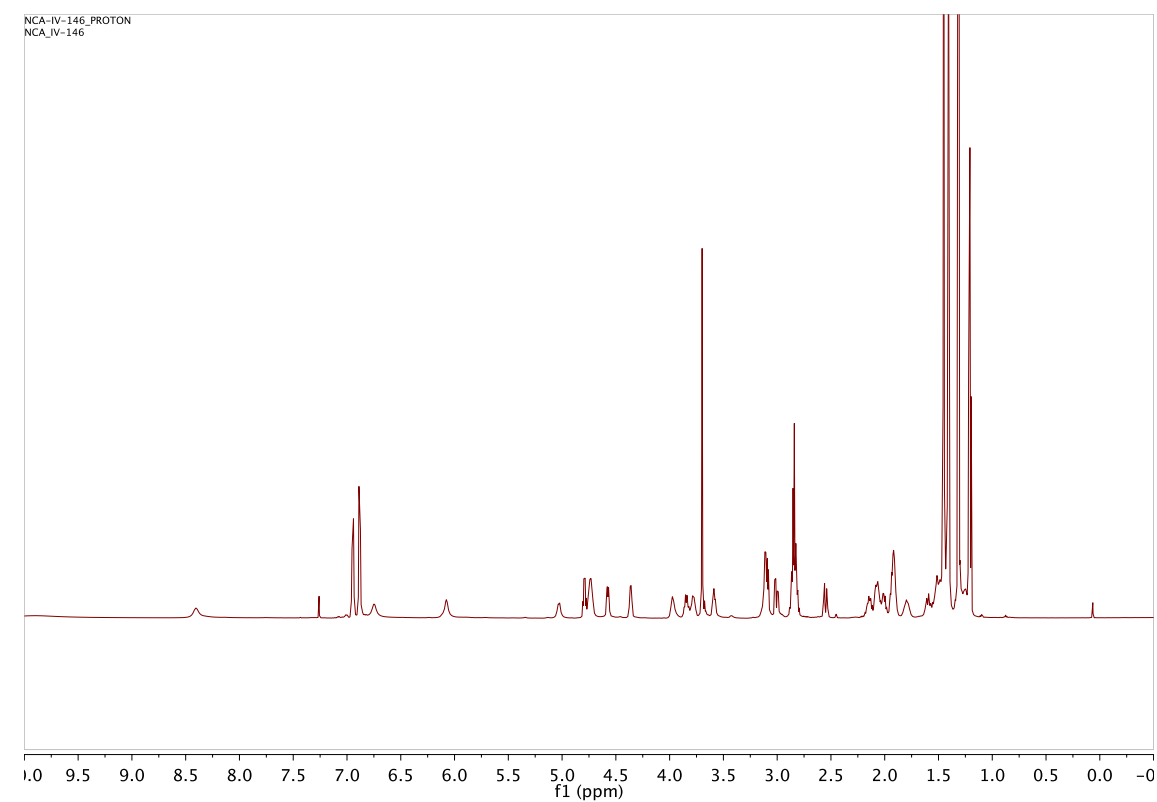


$\cos Y$

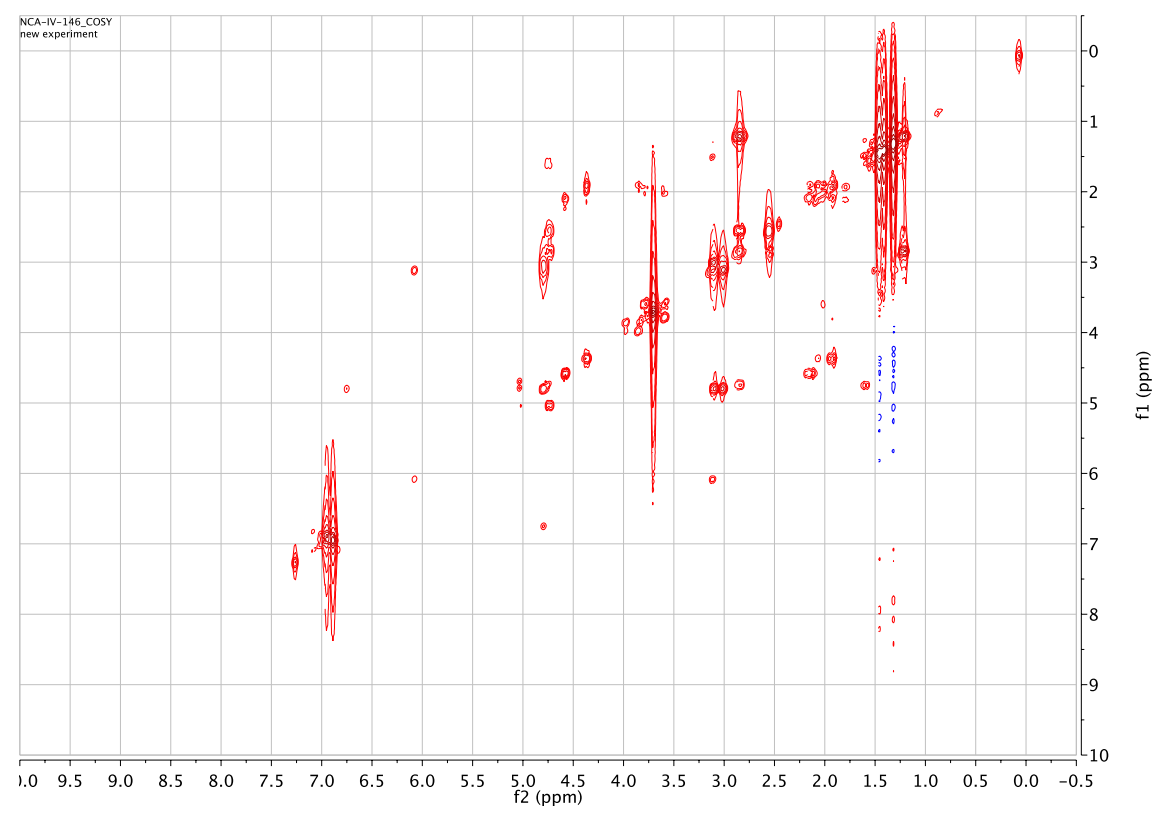

ROESY

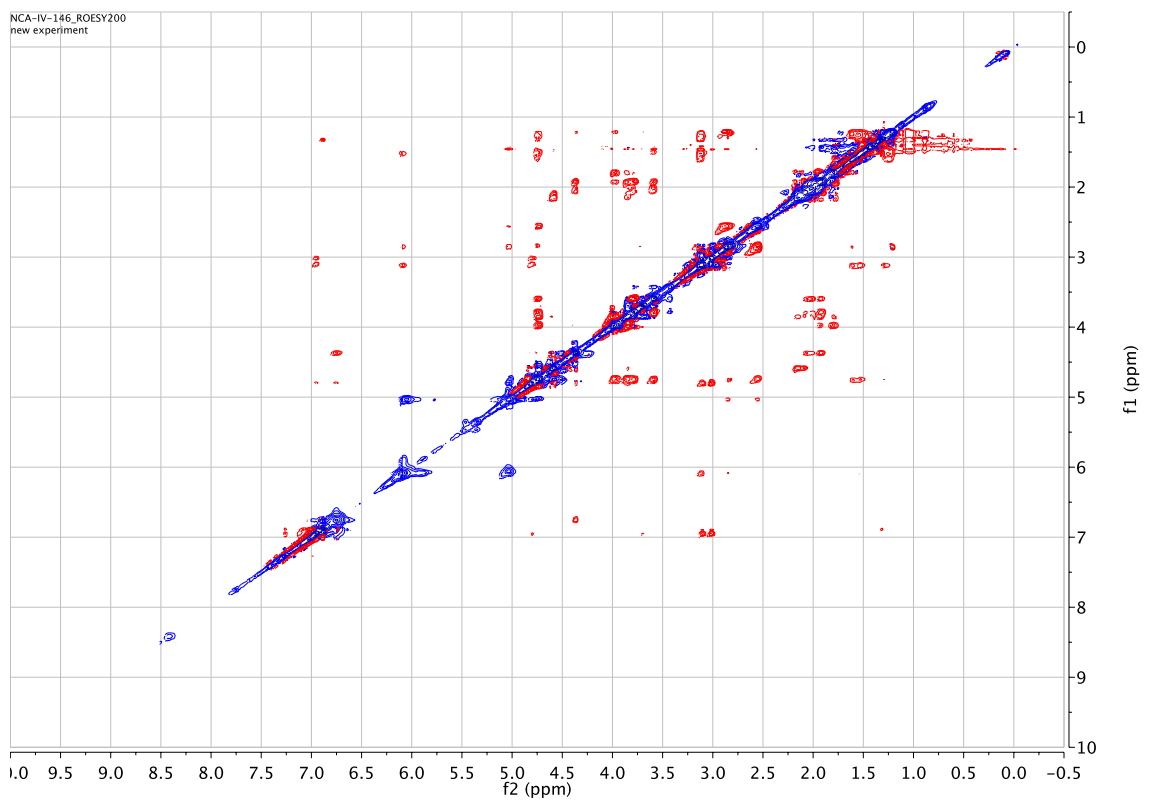

S4 


\section{Peptide 1 + 6}

\section{${ }^{1}$ H NMR}
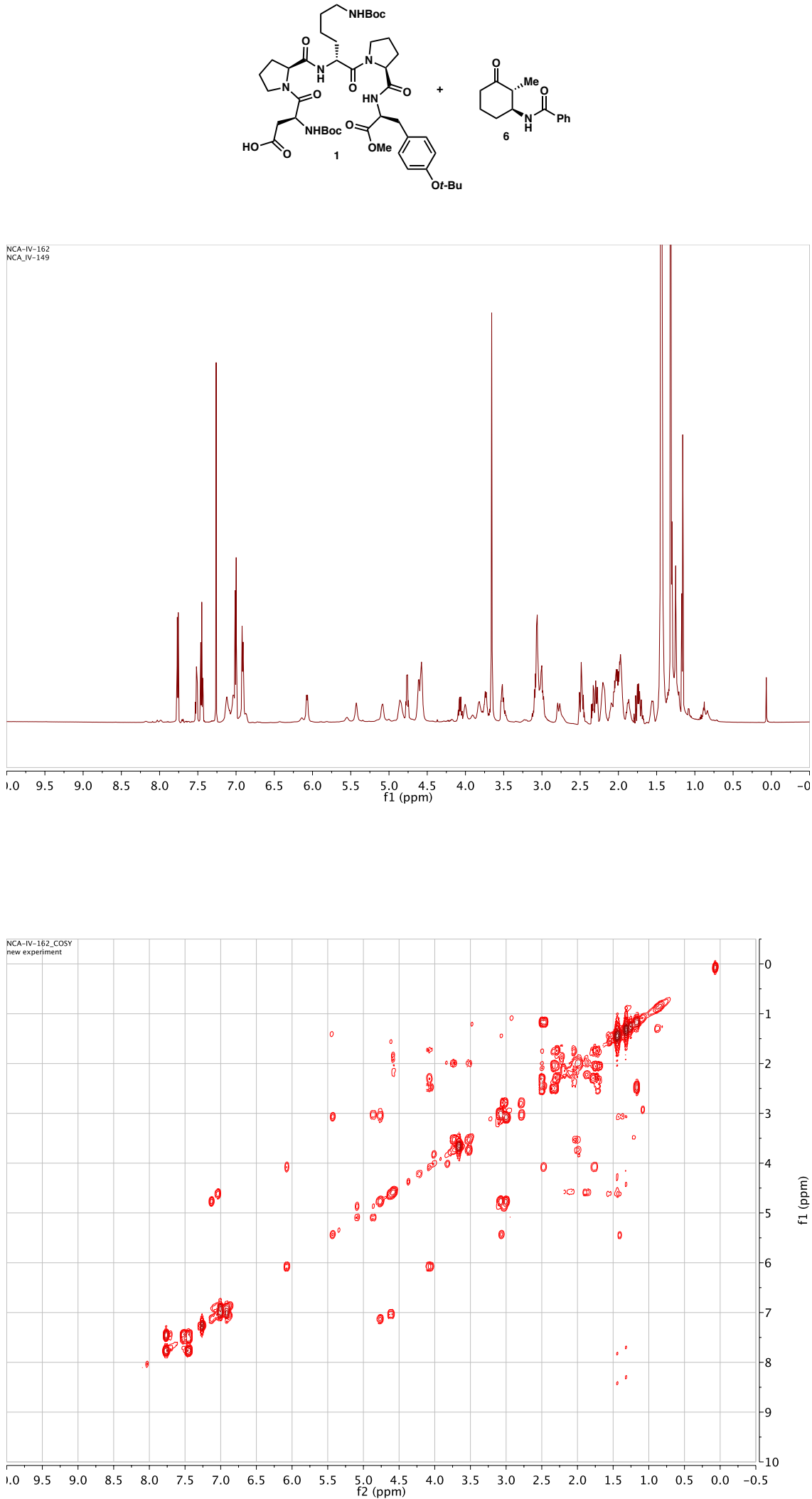


\section{ROESY}

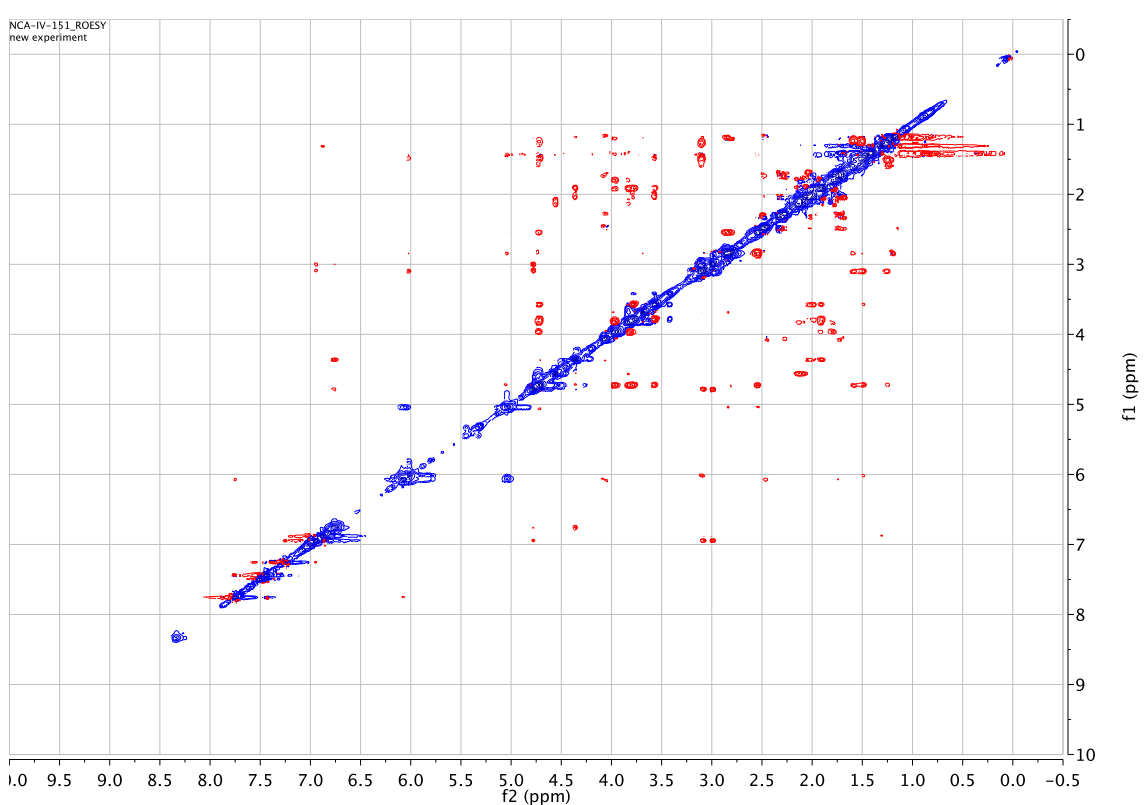




\section{Peptide 1 + 8}

\section{${ }^{1}$ H NMR}
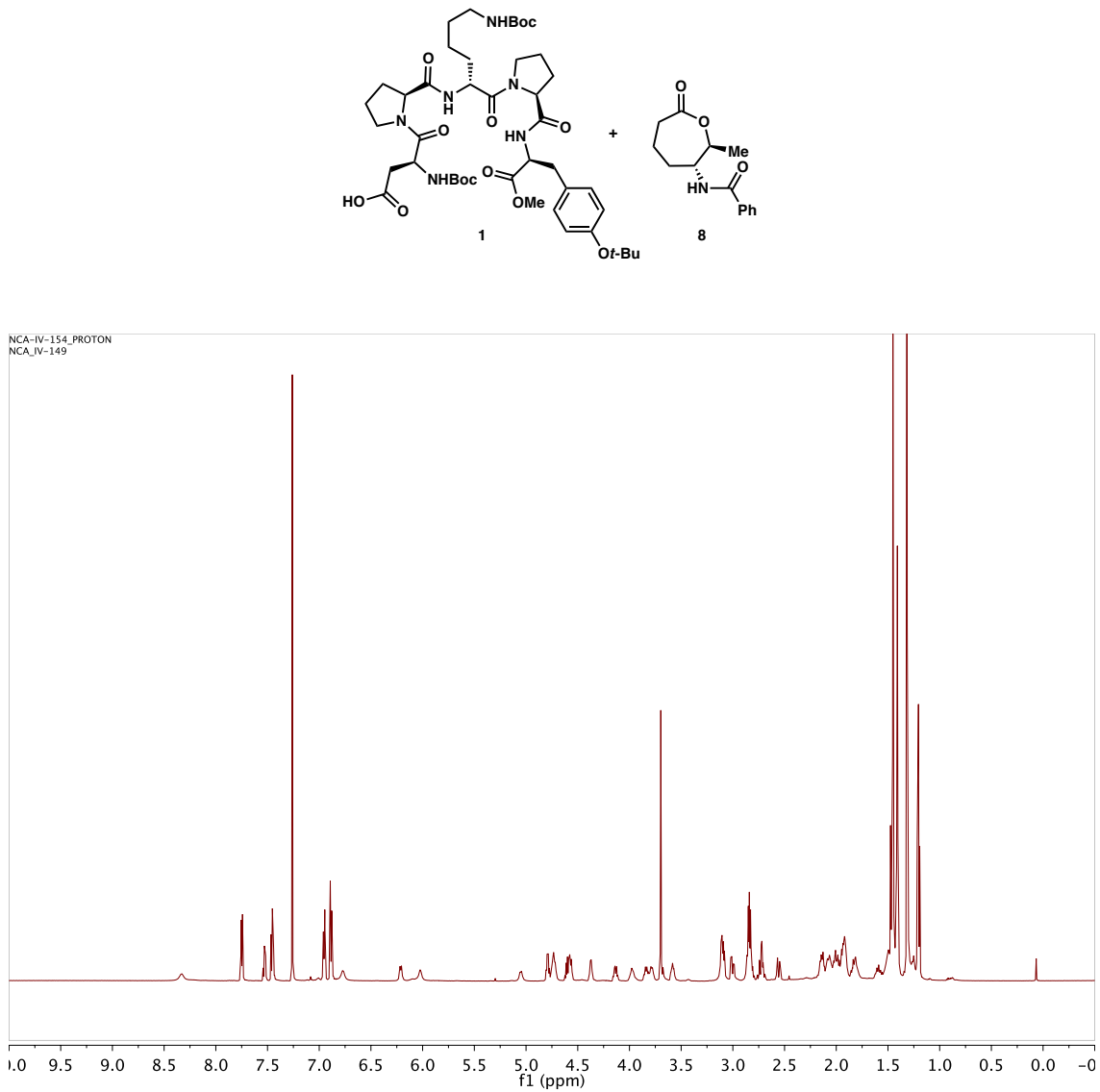

cosy

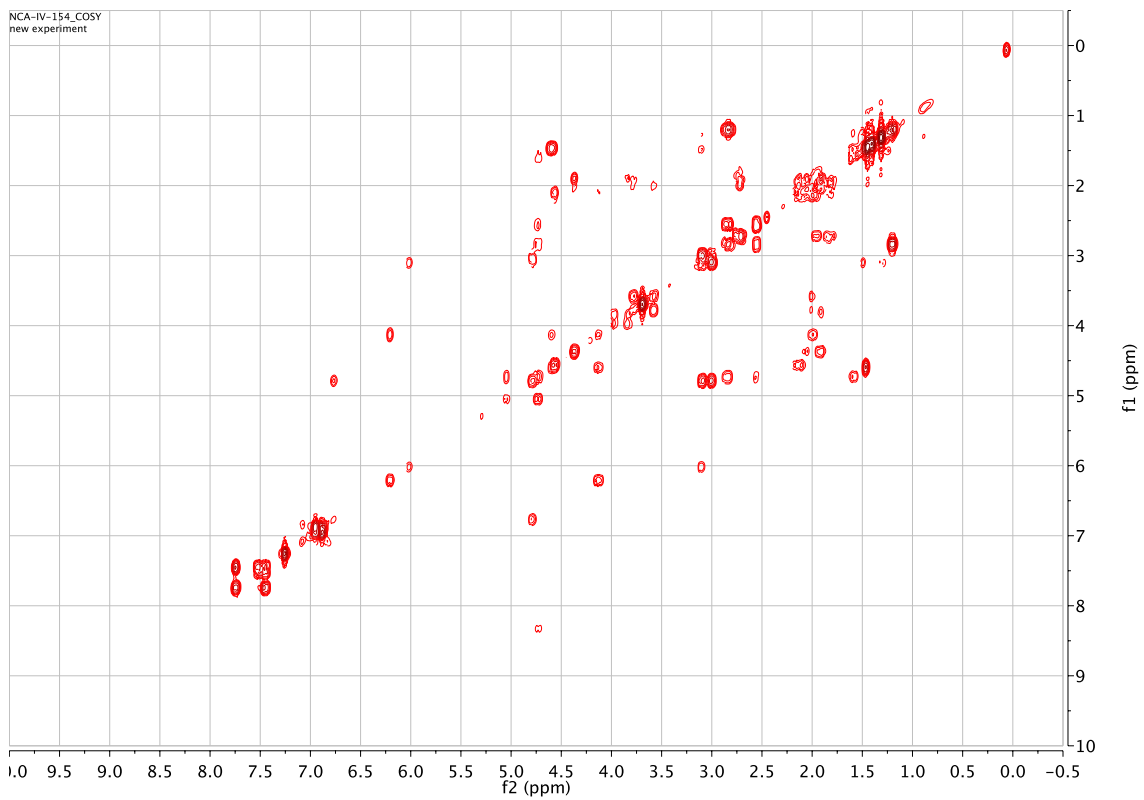




\section{ROESY}

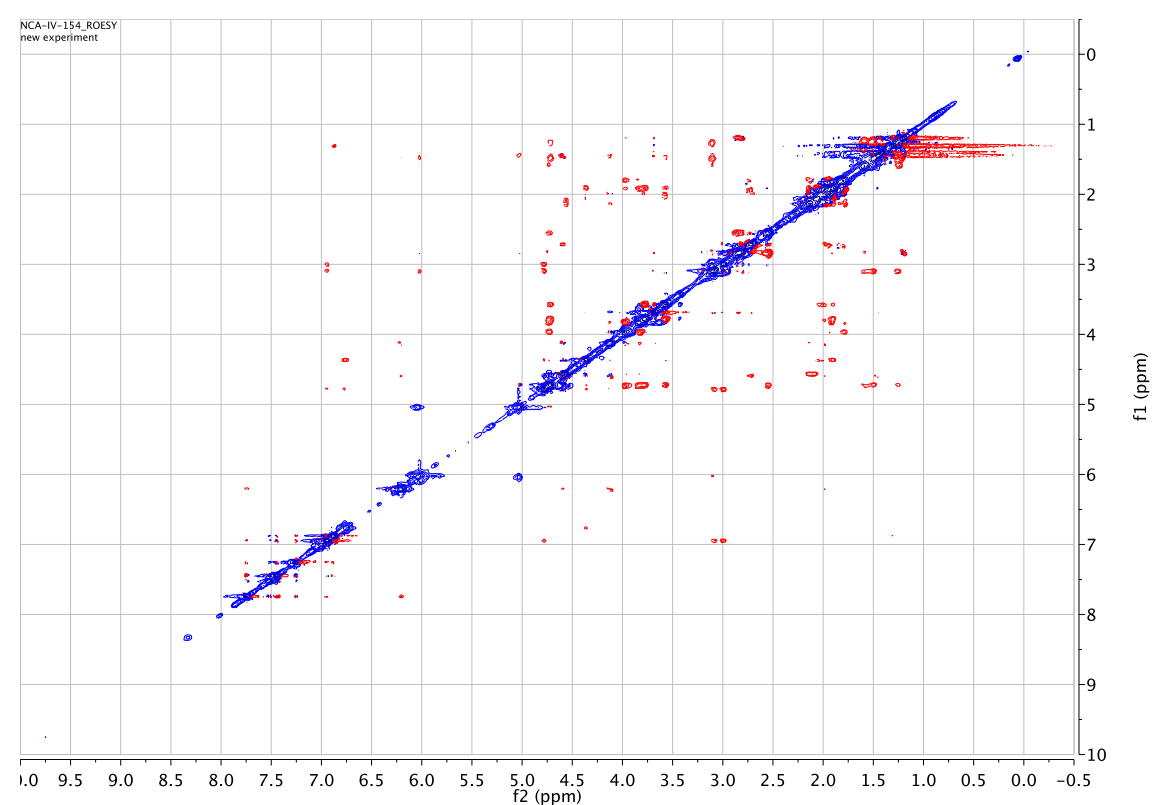

ent $1+6$

${ }^{1}$ H NMR
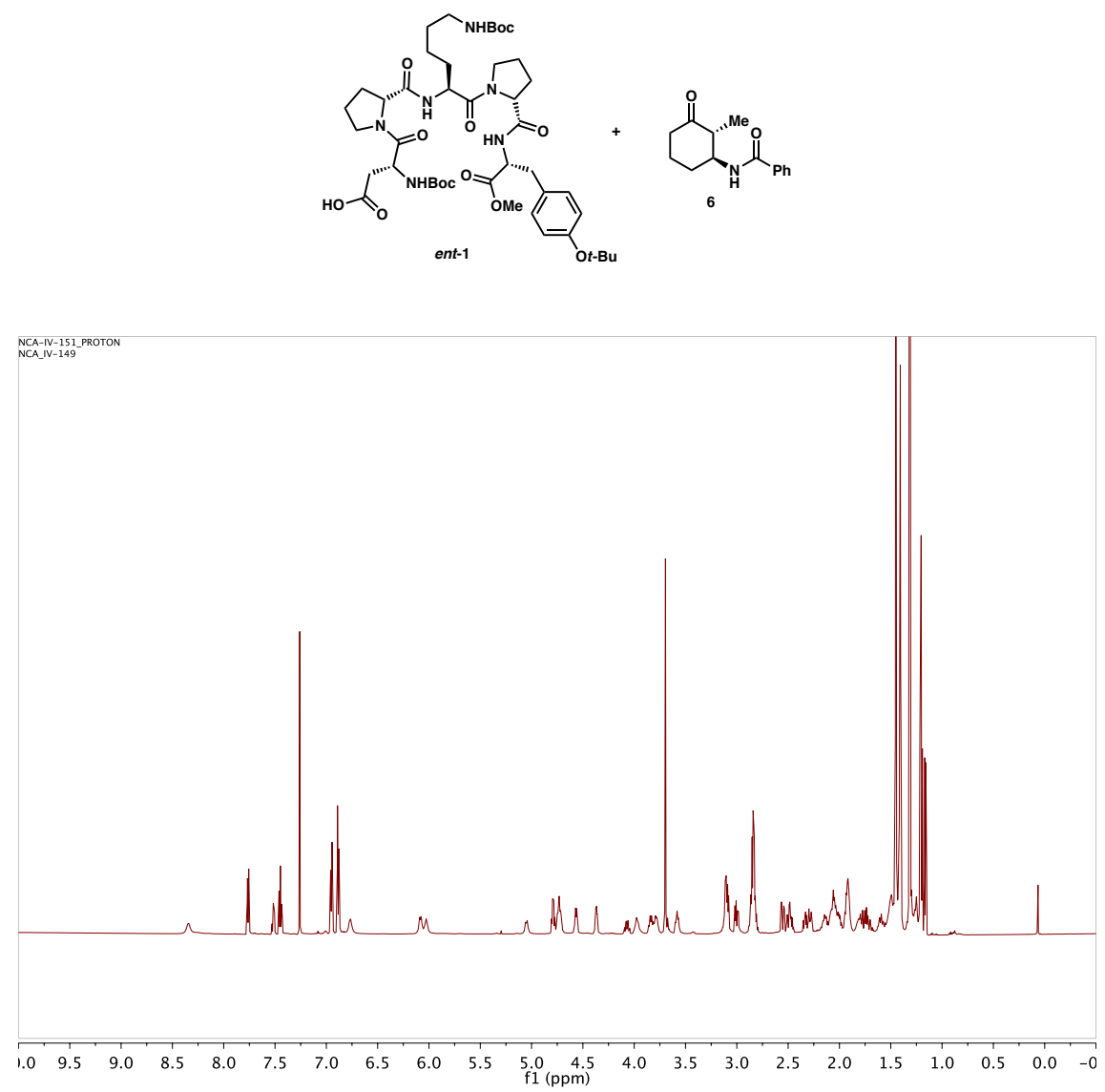

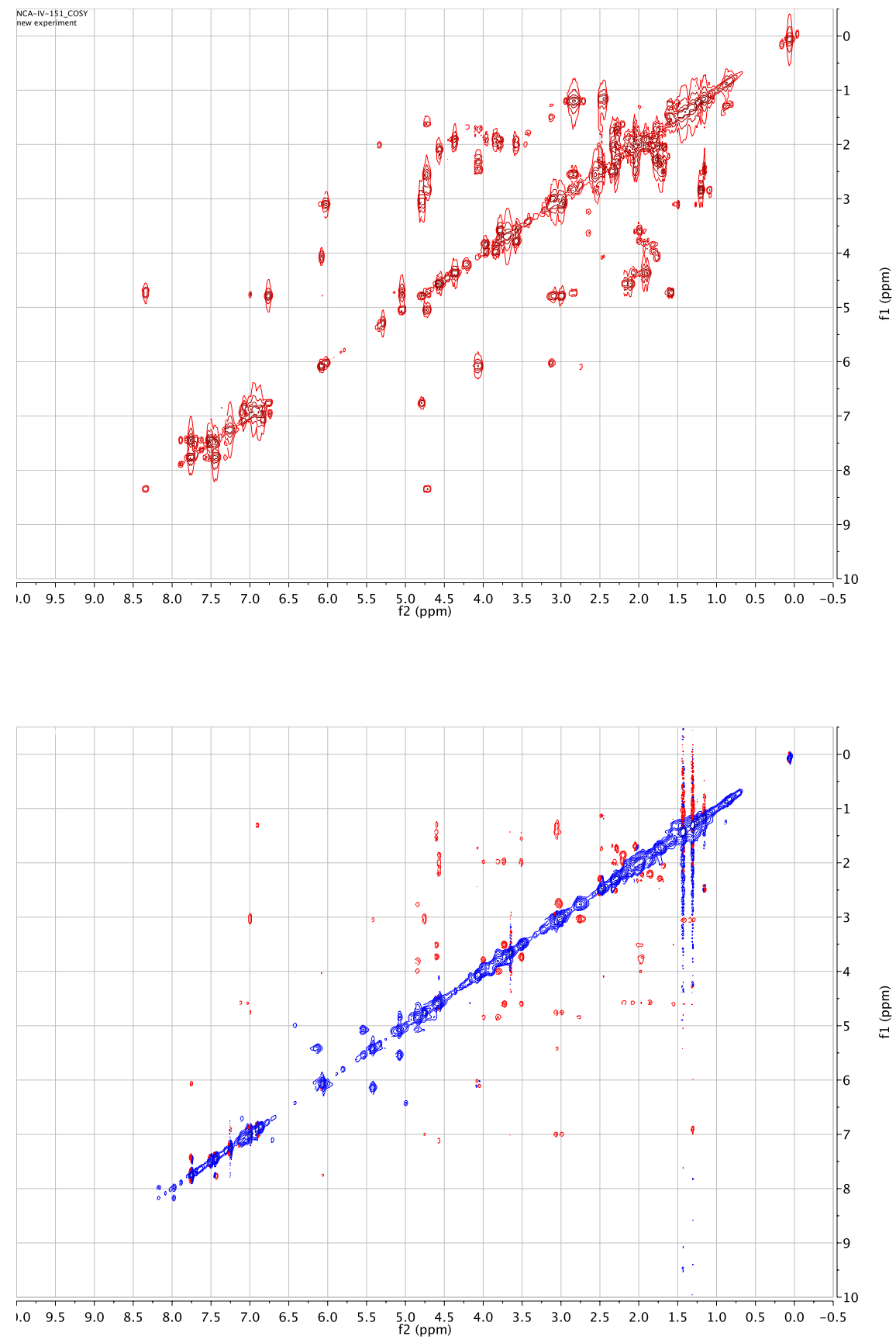

\section{NMR Methods}

To fully characterize peptides in solution, one-dimensional ${ }^{1} \mathrm{H}$ and twodimensional gCOSY and ROESY experiments were carried out for peptide $\mathbf{4}$ and mixed samples of 4. All data were collected on Varian Inova $600 \mathrm{MHz}$ spectrometers that were equipped with $\mathrm{VnmrJ}$, version 4.2 revision $A$. Varian provided the pulse sequences for all experiments. All samples were prepared in $\mathrm{CDCl}_{3}$ (with $\mathrm{CDCl}_{3}$ set to $\left.7.26 \mathrm{ppm}\right)^{1}$ at a concentration of $0.01 \mathrm{M}$. 
The ROESY for each sample was collected at ambient temperature with a mixing time of $200 \mathrm{~ms}$, a spectral width of $5459 \mathrm{~Hz}$, and a d1 time of $3.5 \mathrm{~s}$. The data was acquired with a total of 256 transients, 1442 points in the f2 dimension, and 256 points in the f1 dimension. The spectra were processed using MestReNova, version 9.0.012821. Zero-filling sized the spectra to 2048 , 2048. Automatic phasing was used in conjunction with manual adjustments. Additionally, apodization was accomplished with a sine square function $\left(90^{\circ}\right)$ in both dimensions. Each spectrum was automatically baseline corrected in each dimension using the Bernstein third order polynomial fit and symmetrized using COSY-like symmetrization. Further refinement included treatment of the spectrum to reduce $\mathrm{t} 1$ noise. The ROESY spectra were re-examined without COSYlike symmetrization and peaks that were deemed to be potential artifacts were discarded.

ROESY spectra were integrated to extract distances from observed throughspace interactions between protons on each peptide. After integrating NOESY crosspeaks, the peaks' volumes were converted to distances using the equation $(\mathrm{SI}-1){ }^{3}$ where $r_{i j}$ is the calculated distance, $r_{\text {ref }}$ is a reference distance, $v_{\text {ref }}$ is the volume of a reference peak, and $v_{i j}$ is the volume of the cross-peak in question. Reference peaks were chosen to be those that corresponded to interactions between $\delta$-protons on the peptide's respective Pro(4) residue. Reference distances that corresponded to these volumes were extracted from the crystal structure of proline-containing proteins.

$$
r_{i j}=r_{r e f} \sqrt[6]{\left(\frac{v_{r e f}}{v_{i j}}\right)}
$$

Integrated volumes were corrected using equation $(\mathrm{SI}-2),{ }^{4}$ where $v$ is the volume corresponding to either the reference or the peak in question from ESI-1, $v_{\text {raw }}$ is the uncorrected volume of a peak in question, and $v_{\text {diag1 }}$ and $v_{\text {diag2 }}$ correspond to the volumes of the diagonal peaks for each respective interacting proton.

$$
v=\frac{2 v_{\text {raw }}}{\left(v_{\text {diag } 1}+v_{\text {diag } 2}\right)}
$$

The restraints were then processed using the standard Crystallography and NMR Systems $(\mathrm{CNS})^{5}$ simulated annealing protocol. A parameter file for each residue was assembled within the program. Each peptide was treated with the macro commands generate_seq, generate_extended, and anneal. By altering the energy-scoring threshold in the program's accept input file, we were able to cull the 10-lowest energy scored conformations for each structure. The accept feature of CNS also generated the average structure of these 10 conformers, which in turn, became the input for DFT calculations. Bins were defined at 1.8 to $2.5 \AA$, 1.8 to $3.0 \AA$, 1.8 to $3.5 \AA$, and 1.8 to 4.5 $\AA$. Distances that were calculated to be over $4.5 \AA$ were not included in the CNS restraint file.

The simulated annealing outputs from CNS were then used as starting geometries for optimization and frequency calculation at the B3LYP/6-31G(d,p) level of theory using Gaussian 09. ${ }^{6,7}$ Chloroform was specified as the implicit solvent using the IEFPCM protocol. ${ }^{8}$ Each structure was restrained using rOe-derived redundant internal coordinates. For all mixed samples, the redundant internal coordinates were chosen to 
be $\mathrm{NH}_{\text {Lys-sidechain }}$ to $\beta_{\text {'Tyr }}$ and $\mathrm{NH}_{\text {Lys-sidechain }}$ to $\beta_{\text {Asp }}$ (see Table $\mathrm{SI-1}$ for specific restraints). For the structure of $\mathbf{4}$ without any additive, $\alpha_{\text {Pro(2) }}$ to $\mathrm{NH}_{\text {Tyr }}$ was restrained to $2.3 \AA, \mathrm{NH}_{\mathrm{Lys}}$ to $\alpha_{\text {Tyr }}$ was restrained to $3.0 \AA$, and $\alpha_{A s p}$ to $\delta^{\prime}$ Pro(2) was restrained to $2.0 \AA$.

Table SI-1

\begin{tabular}{|c|c|c|}
\hline Sample & $\begin{array}{c}\mathrm{NH}_{\text {Lys-sidechain }} \text { to } \beta^{\prime}{ }^{\prime}{ }_{\text {Tyr }} \\
\text { distance }(\AA)\end{array}$ & $\begin{array}{c}\text { NH }_{\text {Lys-sidechain }} \text { to } \beta^{\prime}{ }_{\text {Asp }} \\
\text { distance }(\AA)\end{array}$ \\
\hline $\mathbf{1 + 6}$ & 2.8 & 3.4 \\
\hline $\mathbf{1 + 8}$ & 2.5 & 2.9 \\
\hline ent-1+6 & 2.6 & 3.4 \\
\hline
\end{tabular}

\section{NMR Solution-Phase Studies}

\section{Peptide 1}
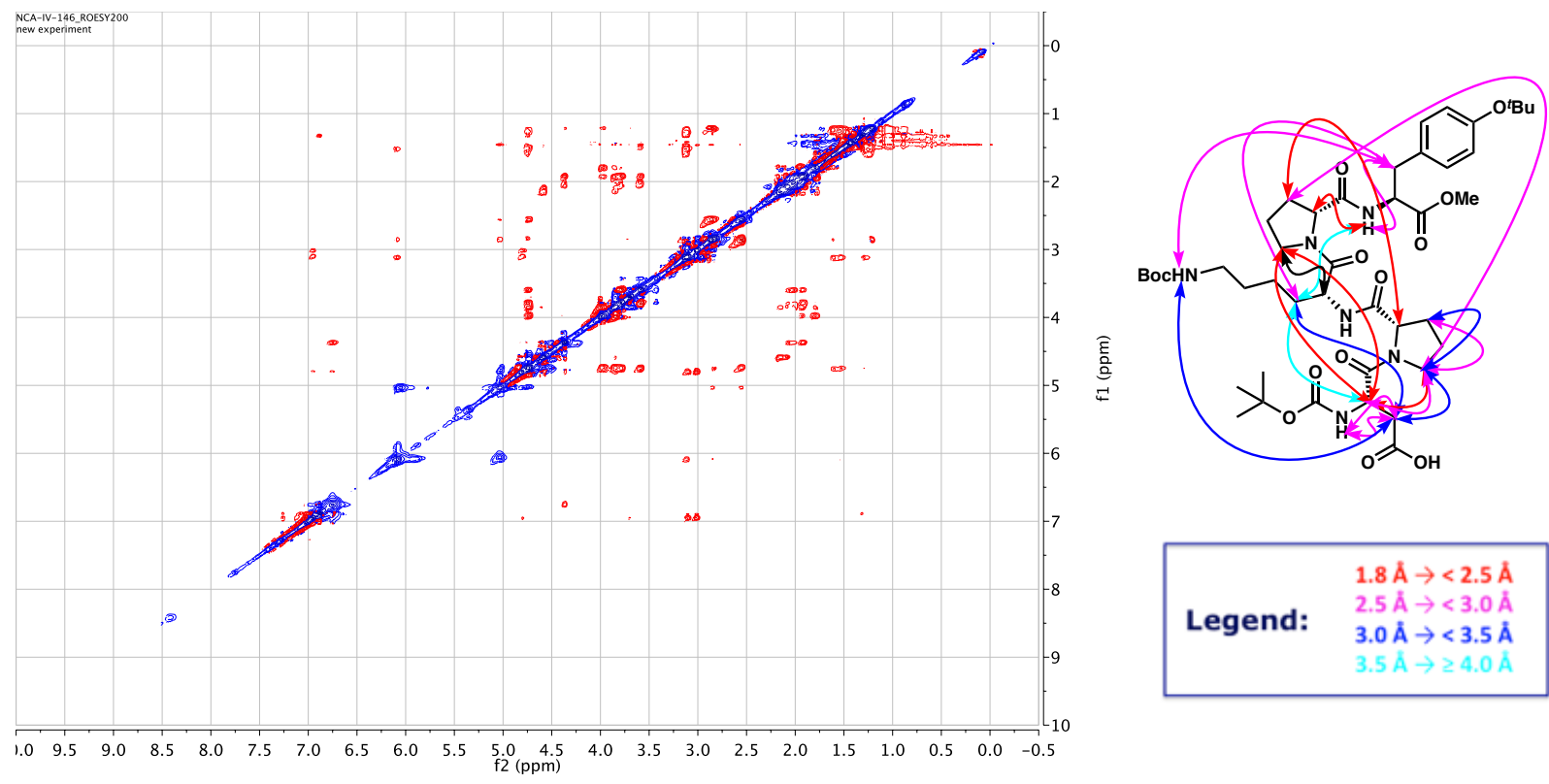

ROESY Contacts

\begin{tabular}{|c|c|c|c|c|c|c|}
\hline & f2 & f1 & Normalized & Absolute & Assignment & $\begin{array}{c}\text { Corrected } \\
\text { Distances } \\
\text { (Normalized) }\end{array}$ \\
\hline \multirow{2}{*}{1} & 6.75 & 3.01 & 0.57 & 658.69 & \multirow{2}{*}{$\begin{array}{l}\text { Tyr(5)NH- } \\
\text { Tyr(5)HB1 }\end{array}$} & \multirow{2}{*}{2.98} \\
\hline & 3.01 & 6.75 & 0.47 & 545.05 & & \\
\hline \multirow{2}{*}{2} & 6.74 & 3.1 & 0.3 & 346.16 & \multirow{2}{*}{ Tyr(5)NH-Lys(3)HB } & \multirow{2}{*}{3.80} \\
\hline & 3.09 & 6.75 & 0.17 & 194.24 & & \\
\hline \multirow{2}{*}{3} & 6.72 & 4.37 & 3.33 & 3862.84 & \multirow{2}{*}{ Tyr(5)NH-Pro(4)HA } & \multirow{2}{*}{2.16} \\
\hline & 4.37 & 6.75 & 2.78 & 3223.27 & & \\
\hline \multirow{2}{*}{4} & 6.08 & 3.12 & 2 & 2314.16 & \multirow{2}{*}{$\begin{array}{c}\text { Lys(3)NRH- } \\
\text { Tyr(5)HB2 }\end{array}$} & \multirow{2}{*}{2.60} \\
\hline & 3.11 & 6.09 & 2.34 & 2713.85 & & \\
\hline
\end{tabular}




\begin{tabular}{|c|c|c|c|c|c|c|}
\hline 5 & $\begin{array}{l}6.08 \\
2.85\end{array}$ & $\begin{array}{l}2.86 \\
6.09\end{array}$ & $\begin{array}{c}1.37 \\
1.2\end{array}$ & $\begin{array}{l}1585.87 \\
1385.26\end{array}$ & $\begin{array}{l}\text { Lys(3)NRH- } \\
\text { Asp(1)HB2 }\end{array}$ & 3.00 \\
\hline \multirow{2}{*}{6} & 5.04 & 2.85 & 1.98 & 2293.74 & \multirow{2}{*}{$\begin{array}{l}\text { Asp(1)NH- } \\
\text { Asp(1)HB2 }\end{array}$} & \multirow{2}{*}{2.88} \\
\hline & 2.84 & 5.04 & 1.45 & 1679.64 & & \\
\hline \multirow{2}{*}{7} & 5.03 & 2.56 & 0.69 & 803.81 & \multirow{2}{*}{$\begin{array}{l}\text { Asp(1)NH- } \\
\text { Asp(1)HB1 }\end{array}$} & \multirow{2}{*}{2.64} \\
\hline & 2.55 & 5.04 & 1.08 & 1255.41 & & \\
\hline \multirow{2}{*}{8} & 4.73 & 3.1 & 0.5 & 584.22 & \multirow{2}{*}{$\begin{array}{l}\text { Asp(1)HA- } \\
\text { Lys(3)HB }\end{array}$} & \multirow{2}{*}{3.53} \\
\hline & 3.1 & 4.72 & 0.57 & 656.48 & & \\
\hline \multirow{2}{*}{9} & 4.73 & 3.76 & 4.78 & 5538.73 & \multirow{2}{*}{$\begin{array}{l}\text { Asp(1)HA- } \\
\text { Pro(4)HD2 }\end{array}$} & \multirow{2}{*}{2.39} \\
\hline & 3.77 & 4.71 & 4.64 & 5378.53 & & \\
\hline \multirow{2}{*}{10} & 4.72 & 3.59 & 3.43 & 3976.95 & \multirow{2}{*}{$\begin{array}{l}\text { Asp(1)HA- } \\
\text { Pro(4)HD1 }\end{array}$} & \multirow{2}{*}{2.43} \\
\hline & 3.59 & 4.72 & 3.12 & 3614.15 & & \\
\hline \multirow{2}{*}{11} & 4.72 & 3.97 & 6.34 & 7343.89 & \multirow{2}{*}{$\begin{array}{l}\text { Asp(1)HA- } \\
\text { Pro(2)HD2 }\end{array}$} & \multirow{2}{*}{2.19} \\
\hline & 3.97 & 4.72 & 6.6 & 7652.33 & & \\
\hline \multirow{2}{*}{12} & 4.57 & 2.07 & 2.25 & 2612.55 & \multirow{2}{*}{ Pro(2)HA-Pro(4)HB } & \multirow{2}{*}{2.43} \\
\hline & 2.09 & 4.59 & 2.85 & 3302.89 & & \\
\hline \multirow{2}{*}{13} & 3.98 & 2.55 & 0.4 & 468.73 & \multirow{2}{*}{$\begin{array}{l}\text { Pro(2)HD2- } \\
\text { Asp(1)HB1 }\end{array}$} & \multirow{2}{*}{3.30} \\
\hline & 2.54 & 3.98 & 0.38 & 445.65 & & \\
\hline \multirow{2}{*}{14} & 3.98 & 2.08 & 0.55 & 636.42 & \multirow{2}{*}{$\begin{array}{l}\text { Pro(2)HD2- } \\
\text { Pro(2)HB1 }\end{array}$} & \multirow{2}{*}{3.26} \\
\hline & 2.08 & 3.98 & 0.38 & 441.16 & & \\
\hline \multirow{2}{*}{15} & 3.84 & 2.08 & 1.08 & 1256.01 & \multirow{2}{*}{$\begin{array}{l}\text { Pro(2)HD1- } \\
\text { Pro(2)HB1 }\end{array}$} & \multirow{2}{*}{2.80} \\
\hline & 2.08 & 3.81 & 1.19 & 1374.76 & & \\
\hline & 3.79 & 3.58 & 19.67 & 22797.3 & \multirow{2}{*}{$\begin{array}{l}\text { Pro(4)HD2- } \\
\text { Pro(4)HD1 }\end{array}$} & 180 \\
\hline & 3.58 & 3.79 & 20.09 & 23283.09 & & 1.80 \\
\hline
\end{tabular}




\section{Peptide $1+6$}
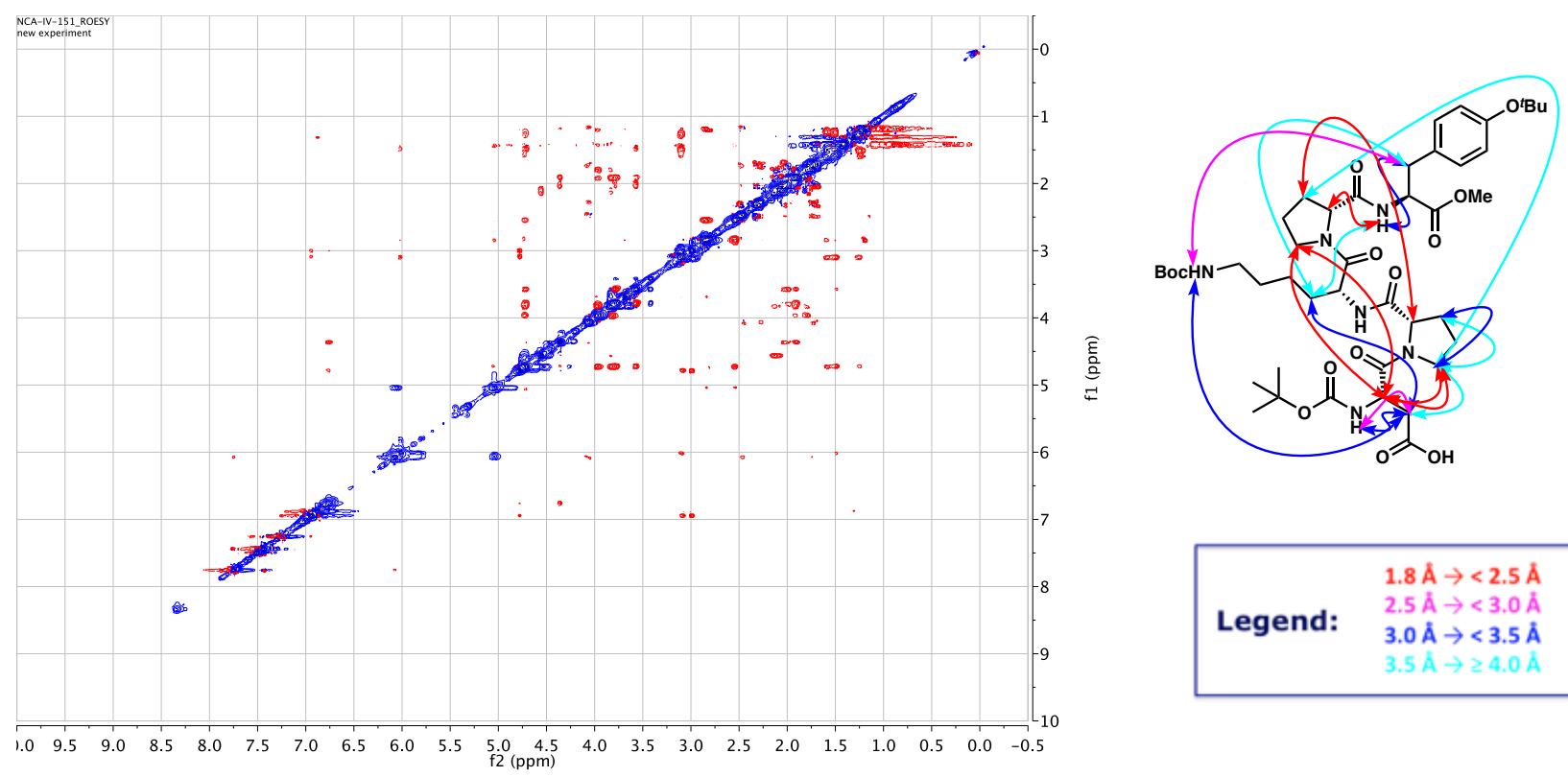

\section{ROESY Contacts}

\begin{tabular}{|c|c|c|c|c|c|c|}
\hline & f2 & $\mathrm{f1}$ & Normalized & Absolute & Assignment & $\begin{array}{c}\text { Corrected } \\
\text { Distances } \\
\text { (Normalized) }\end{array}$ \\
\hline \multirow{2}{*}{1} & 6.76 & 2.99 & 0.16 & 467.14 & Tyr(5)NH- & \multirow{2}{*}{3.03} \\
\hline & 2.99 & 6.75 & 0.15 & 433.54 & Tyr(5)HB1 & \\
\hline \multirow{2}{*}{2} & 6.75 & 3.09 & 0.14 & 403.73 & \multirow{2}{*}{ Tyr(5)NH-Lys(3)HB } & \multirow{2}{*}{3.64} \\
\hline & 3.09 & 6.75 & 0.09 & 254.33 & & \\
\hline \multirow{2}{*}{3} & 6.76 & 4.35 & 1 & 2888.36 & \multirow{2}{*}{ Tyr(5)NH-Pro(4)HA } & \multirow{2}{*}{2.26} \\
\hline & 4.36 & 6.75 & 0.63 & 1828.51 & & \\
\hline \multirow{2}{*}{4} & 6.03 & 3.09 & 0.47 & 1367.03 & \multirow{2}{*}{$\begin{array}{c}\text { Lys(3)NRH- } \\
\text { Tyr(5)HB2 }\end{array}$} & \multirow{2}{*}{2.75} \\
\hline & 3.1 & 6.01 & 0.7 & 2013.73 & & \\
\hline \multirow{2}{*}{5} & 6.02 & 2.83 & 0.21 & 617.69 & \multirow{2}{*}{$\begin{array}{l}\text { Lys(3)NRH- } \\
\text { Asp(1)HB2 }\end{array}$} & \multirow{2}{*}{3.38} \\
\hline & 2.84 & 6.02 & 0.19 & 560.89 & & \\
\hline \multirow{2}{*}{6} & 5.04 & 2.83 & 0.49 & 1403.1 & \multirow{2}{*}{$\begin{array}{l}\text { Asp(1)NH- } \\
\text { Asp(1)HB2 }\end{array}$} & \multirow{2}{*}{2.96} \\
\hline & 2.84 & 5.04 & 0.43 & 1246.92 & & \\
\hline \multirow{2}{*}{7} & 5.04 & 2.53 & 0.19 & 534.82 & \multirow{2}{*}{$\begin{array}{l}\text { Asp(1)HN- } \\
\text { Asp(1)HB1 }\end{array}$} & \multirow{2}{*}{2.89} \\
\hline & 2.55 & 5.03 & 0.2 & 567.28 & & \\
\hline \multirow{2}{*}{8} & 4.72 & 3.76 & 2.02 & 5824.2 & \multirow{2}{*}{$\begin{array}{l}\text { Asp(1)HA- } \\
\text { Pro(4)HD2 }\end{array}$} & \multirow{2}{*}{2.21} \\
\hline & 3.77 & 4.72 & 2.81 & 8106.36 & & \\
\hline \multirow{2}{*}{9} & 4.72 & 3.56 & 1.45 & 4181.39 & \multirow{2}{*}{$\begin{array}{l}\text { Asp(1)HA- } \\
\text { Pro(4)HD1 }\end{array}$} & \multirow{2}{*}{2.36} \\
\hline & 3.57 & 4.71 & 1.93 & 5573.23 & & \\
\hline \multirow{2}{*}{10} & 4.72 & 3.97 & 1.82 & 5257.93 & \multirow{2}{*}{$\begin{array}{l}\text { Asp(1)HA- } \\
\text { Pro(2)HD2 }\end{array}$} & \multirow{2}{*}{2.26} \\
\hline & 3.96 & 4.72 & 2.55 & 7350.9 & & \\
\hline \multirow{2}{*}{11} & 4.56 & 2.05 & 0.58 & 1681.72 & \multirow{2}{*}{ Pro(2)HA-Pro(4)HB } & \multirow{2}{*}{2.53} \\
\hline & 2.06 & 4.55 & 0.71 & 2053.46 & & \\
\hline
\end{tabular}




\begin{tabular}{|ccccccc}
12 & 3.98 & 2.54 & 0.09 & 262.09 & Pro(2)HD2- & 3.67 \\
& 2.56 & 3.97 & 0.07 & 205.16 & Asp(1)HB1 & \\
13 & 3.97 & 2.07 & 0.09 & 272.82 & Pro(2)HD2- & 3.57 \\
& 2.08 & 3.97 & 0.09 & 251.08 & Pro(2)HB1 & \\
14 & 3.83 & 2.05 & 0.2 & 591.61 & Pro(2)HD1- & 3.07 \\
& 2.08 & 3.82 & 0.17 & 504.22 & Pro(2)HB1 & \\
& & & & & & \\
& 3.78 & 3.55 & 5.8 & 16754.79 & Pro(4)HD2- & \\
\hline
\end{tabular}

\section{Peptide $1+8$}

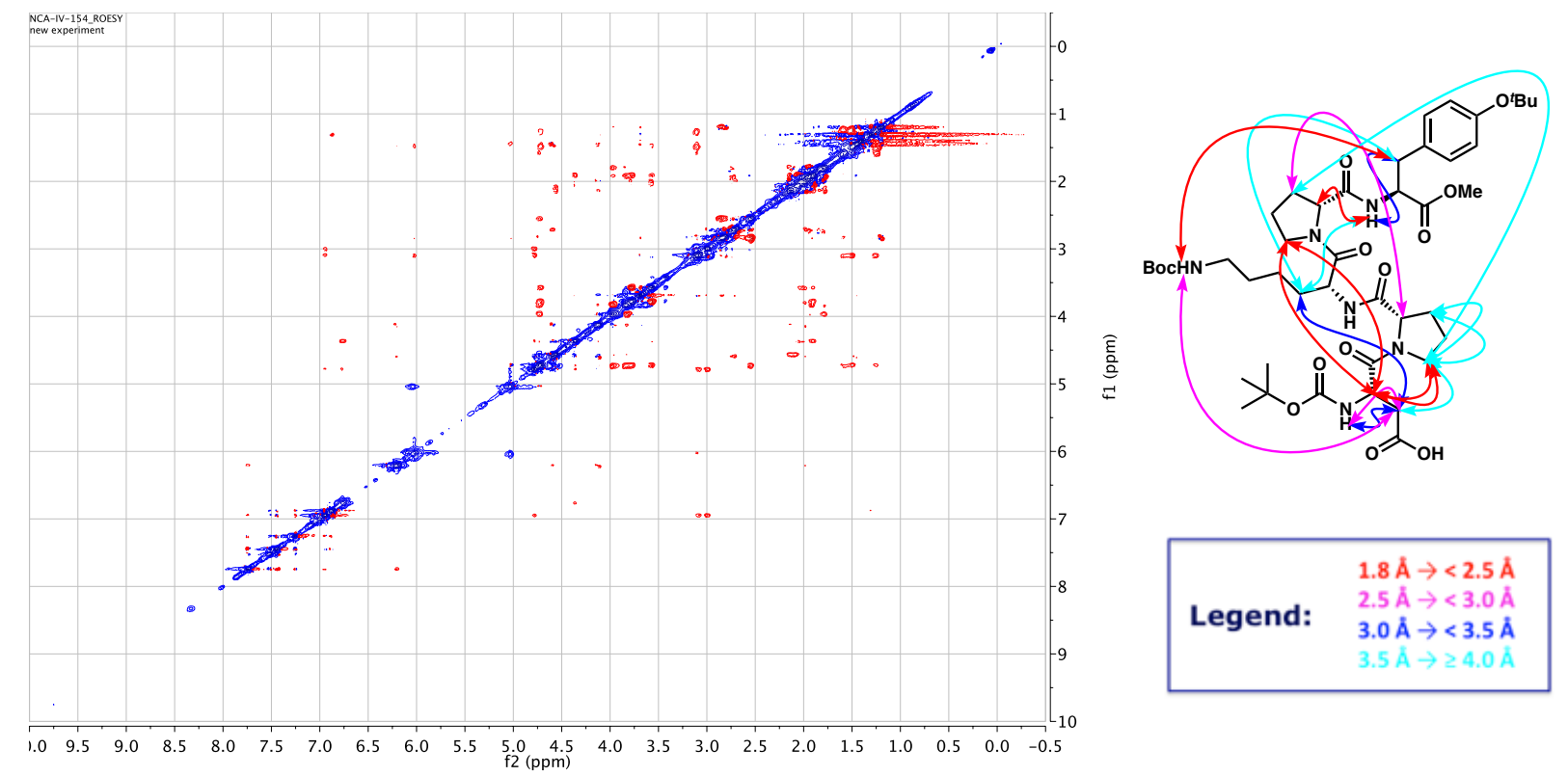

ROESY Contacts

f2 f1
Normalized Absolute Assignment

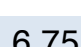

6.75

2.98

6.76

2

3

4

5

6

7
3.08

6.76

4.35

6.03

3.1

6.01

2.85

5.03

2.86

5.03

2.97
6.76
3.09
6.77
4.36
6.78
3.09
6.01
2.84
6.01
2.85
5.04
2.55

2.55

$\begin{array}{rr}0.05 & 123.26 \\ 0.05 & 127.75 \\ 0.07 & 170.3 \\ 0.03 & 74.18 \\ 0.92 & 2257.31 \\ 0.42 & 1024.95 \\ 0.47 & 1367.03 \\ 0.7 & 2013.73 \\ 0.2 & 482.35 \\ 0.19 & 472.16 \\ 0.12 & 297.64 \\ 0.15 & 364.5 \\ 0.15 & 374.57\end{array}$

$\operatorname{Tyr}(5) \mathrm{NH}-$ Tyr(5)HB1

Tyr(5)NHLys(3)HB

Tyr(5)NHPro(4)HA

Lys(3)NRHTyr(5)HB2

Lys(3)NRHAsp(1)HB2

Corrected Distances

(Normalized) 


\begin{tabular}{|c|c|c|c|c|c|c|}
\hline & 2.54 & 5.04 & 0.14 & 351.08 & Asp(1)HB1 & \\
\hline \multirow{2}{*}{8} & 4.72 & 3.76 & 1.53 & 3764.6 & Lys(3)HA- & \multirow{2}{*}{2.21} \\
\hline & 3.77 & 4.73 & 2.19 & 5384.1 & Pro(4)HD1 & \\
\hline \multirow{2}{*}{9} & 4.72 & 3.57 & 1.09 & 2684.17 & Asp(1)HA- & \multirow{2}{*}{2.36} \\
\hline & 3.57 & 4.71 & 1.48 & 3629.59 & Pro(4)HD2 & \\
\hline \multirow{2}{*}{10} & 4.73 & 3.96 & 1.36 & 3352.28 & Lys(3)HA- & \multirow{2}{*}{2.24} \\
\hline & 3.96 & 4.73 & 2.04 & 5008.3 & Pro(2)HD2 & \\
\hline \multirow{2}{*}{11} & 4.56 & 2.06 & 0.47 & 1154.49 & Pro(2)HA- & \multirow{2}{*}{2.74} \\
\hline & 2.05 & 4.56 & 0.4 & 990.85 & Pro(4)HB2 & \\
\hline \multirow{2}{*}{12} & 3.98 & 2.54 & 0.09 & 262.09 & Pro(2)HD2- & \multirow{2}{*}{3.53} \\
\hline & 2.56 & 3.97 & 0.07 & 205.16 & Asp(1)HB1 & \\
\hline \multirow{2}{*}{13} & 3.97 & 2.07 & 0.09 & 272.82 & Pro(2)HD2- & \multirow{2}{*}{3.55} \\
\hline & 2.08 & 3.97 & 0.09 & 251.08 & Pro(4)HB1 & \\
\hline \multirow{2}{*}{14} & 3.84 & 2.07 & 0.11 & 277.77 & Pro(2)HD1- & \multirow{2}{*}{3.70} \\
\hline & 2.03 & 3.84 & 0.02 & 54.4 & Pro(4)HB1 & \\
\hline & 3.78 & 3.56 & 4.57 & 11237.08 & Pro(4)HD2- & \multirow{2}{*}{1.80} \\
\hline & 3.56 & 3.78 & 5.12 & 12590.76 & Pro(4)HD1 & \\
\hline
\end{tabular}

ent-1 + 6

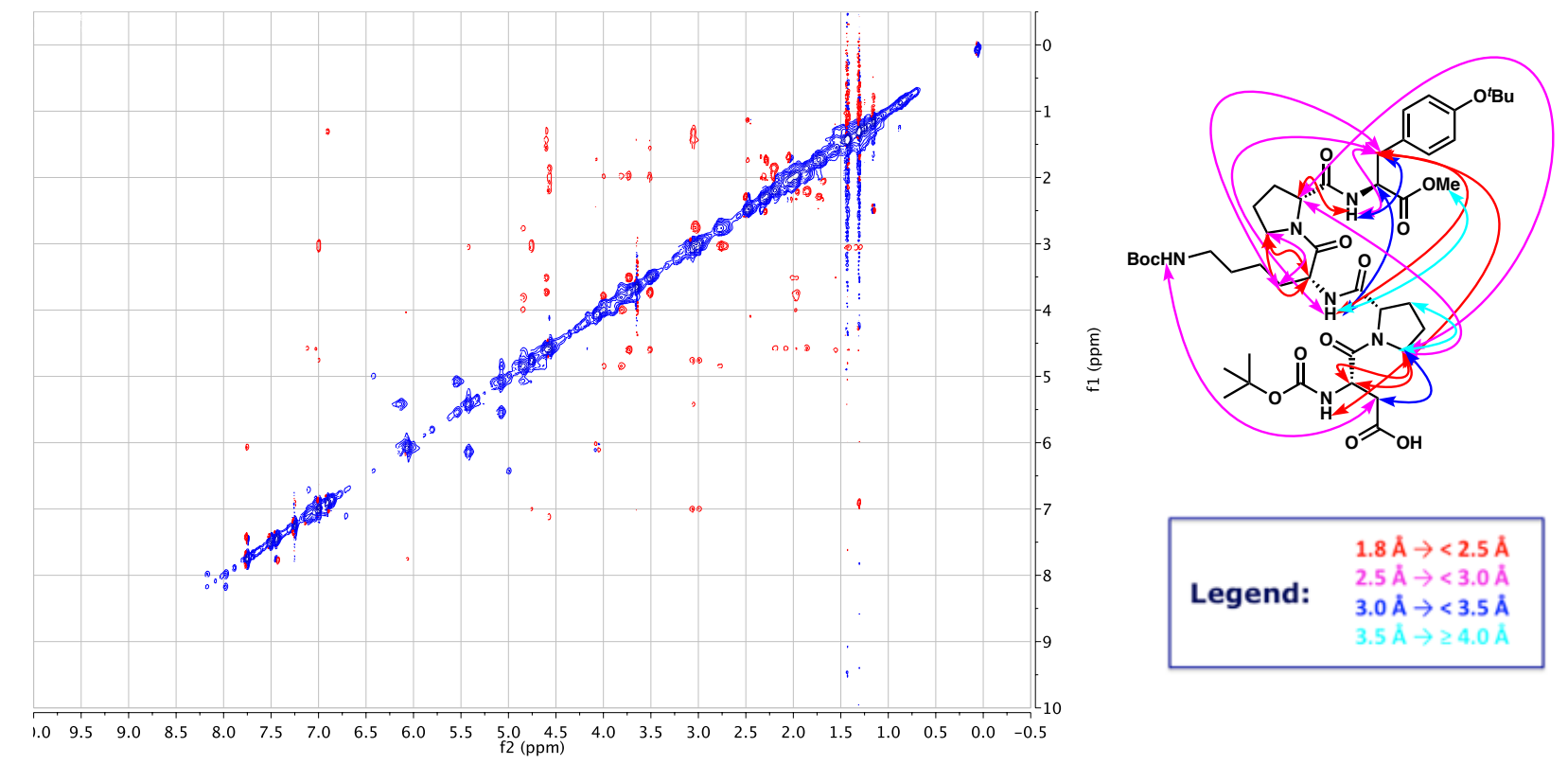

\section{ROESY Contacts}

\begin{tabular}{ccccccc} 
& $f 2$ & $f 1$ & Normalized & Absolute & Assignment & $\begin{array}{c}\text { Corrected } \\
\text { Distances } \\
\text { (Normalized) }\end{array}$ \\
\hline 1 & 7.13 & 3 & 39.85 & 752.52 & $\operatorname{Tyr}(5) \mathrm{NH}-$ & 2.58 \\
2 & 2.99 & 7.12 & 40.49 & 764.63 & $\operatorname{Tyr}(5) \mathrm{HB} 1$ & 3.22
\end{tabular}




\begin{tabular}{|c|c|c|c|c|c|c|}
\hline & 3.09 & 7.11 & 21.55 & 407 & Tyr(5)HB2 & \\
\hline \multirow{2}{*}{3} & 7.12 & 4.58 & 202.92 & 3832.31 & \multirow{2}{*}{ Tyr(5)NH-Pro(2)HA } & \multirow{2}{*}{2.33} \\
\hline & 4.58 & 7.13 & 110.58 & 2088.51 & & \\
\hline \multirow{2}{*}{4} & 7.01 & 4.76 & 35.69 & 673.97 & \multirow{2}{*}{ Lys(3) NH-Tyr(5)HA } & \multirow{2}{*}{2.97} \\
\hline & 4.76 & 7.01 & 62.78 & 1185.61 & & \\
\hline \multirow{2}{*}{5} & 7.01 & 3 & 77.25 & 1458.89 & \multirow{2}{*}{$\begin{array}{l}\text { Lys(3)NH- } \\
\text { Tyr(5)HB1 }\end{array}$} & \multirow{2}{*}{2.49} \\
\hline & 2.99 & 7.01 & 160.09 & 3023.4 & & \\
\hline \multirow{2}{*}{6} & 7.01 & 3.08 & 83.74 & 1581.49 & \multirow{2}{*}{$\begin{array}{l}\text { Lys(3)NH- } \\
\text { Tyr(5)HB2 }\end{array}$} & \multirow{2}{*}{2.76} \\
\hline & 3.08 & 7 & 89.4 & 1688.38 & & \\
\hline \multirow{2}{*}{7} & 7 & 3.66 & 9.52 & 179.76 & \multirow{2}{*}{ Lys(3)NH-OCH3 } & \multirow{2}{*}{3.95} \\
\hline & 3.65 & 7 & 6.74 & 127.36 & & \\
\hline \multirow{2}{*}{8} & 5.41 & 3.05 & 127.83 & 2414.14 & \multirow{2}{*}{$\begin{array}{l}\text { Lys(3)NRH- } \\
\text { Asp(1)HB2 }\end{array}$} & \multirow{2}{*}{2.53} \\
\hline & 3.06 & 5.42 & 106.39 & 2009.26 & & \\
\hline \multirow{2}{*}{9} & 5.09 & 3.02 & 83.4 & 1575.08 & \multirow{2}{*}{$\begin{array}{l}\text { Asp(1)NH- } \\
\text { Tyr(5)HB1 }\end{array}$} & \multirow{2}{*}{2.47} \\
\hline & 3.01 & 5.09 & 79.54 & 1502.17 & & \\
\hline \multirow{2}{*}{10} & 4.85 & 3.8 & 603.21 & 11392.35 & \multirow{2}{*}{$\begin{array}{l}\text { Asp(1)HA- } \\
\text { Pro(2)HD2 }\end{array}$} & \multirow{2}{*}{1.95} \\
\hline & 3.81 & 4.84 & 793.5 & 14986.18 & & \\
\hline \multirow{2}{*}{11} & 4.85 & 4.01 & 285.15 & 5385.33 & \multirow{2}{*}{$\begin{array}{l}\text { Asp(1)HA- } \\
\text { Pro(2)HD1 }\end{array}$} & \multirow{2}{*}{2.23} \\
\hline & 3.99 & 4.86 & 416.66 & 7869.15 & & \\
\hline 12 & 4.62 & 3.74 & 209.85 & 3963.28 & Lys(3)HA- & \\
\hline 12 & 3.73 & 4.62 & 474.06 & 8953.08 & Pro(4)HD2 & 2.29 \\
\hline & 4.62 & 3.52 & 126.7 & 2392.95 & Lys(3)HA- & \\
\hline 13 & 3.52 & 4.63 & 219.24 & 4140.64 & Pro(4)HD1 & 2.53 \\
\hline 11 & 4.58 & 3.75 & 113.91 & 2151.33 & Pro(2)HA- & \\
\hline 14 & 3.74 & 4.56 & 85.72 & 1618.99 & Pro(4)HD2 & 2.15 \\
\hline 15 & 4.58 & 3.52 & 65.2 & 1231.45 & Pro(2)HA- & 270 \\
\hline 10 & 3.51 & 4.57 & 98.05 & 1851.76 & Pro(4)HD1 & 2.19 \\
\hline & 4.01 & 2.19 & 25.43 & 480.23 & Pro(2)HD2- & \\
\hline 16 & 2.19 & 4.01 & 19.17 & 362.14 & Pro(2)HB2 & 3.61 \\
\hline 17 & 4 & 2.78 & 41.4 & 781.82 & Pro(2)HD2- & \\
\hline 17 & 2.78 & 4.01 & 37.6 & 710.1 & Asp(1)HB1 & 3.10 \\
\hline 18 & 3.53 & 1.57 & 114.27 & 2158.17 & Pro(4)HD1- & 258 \\
\hline 10 & 1.57 & 3.52 & 123.11 & 2325 & Lys(3)HB & 2.50 \\
\hline 10 & 3.08 & 1.55 & 41.71 & 787.78 & Tyr(5)HB2- & \\
\hline 19 & 1.56 & 3.05 & 102.87 & 1942.79 & Lys(3)HB & 2.82 \\
\hline & 3.74 & 3.52 & 1351.29 & 25520.65 & Pro(4)HD2- & 180 \\
\hline & 3.52 & 3.75 & 1284.38 & 24257.04 & Pro(4)HD1 & 1.80 \\
\hline
\end{tabular}


Ten Lowest-Energy Scored Structures from Simulated Annealing with CNS

\section{Peptide 1}

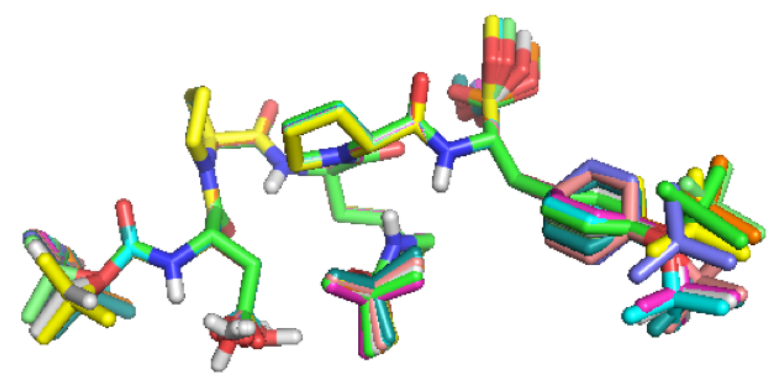

\begin{tabular}{ccc}
\hline Residue & Back & Side \\
\hline Boc & 0.010 & 1.221 \\
Asp & 0.015 & 0.685 \\
Pro & 0.023 & 0.034 \\
Lys & 0.009 & 0.359 \\
DPro & 0.017 & 0.030 \\
Tyr(t-Bu) & 0.021 & 1.309 \\
OMe & 0.000 & 1.304 \\
\hline
\end{tabular}

Peptide $1+6$

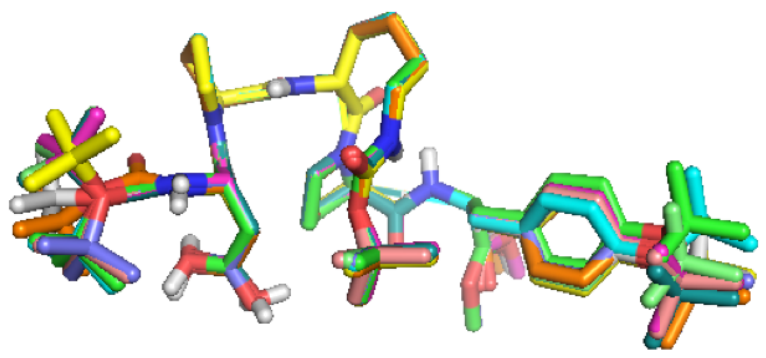

Peptide $1+8$

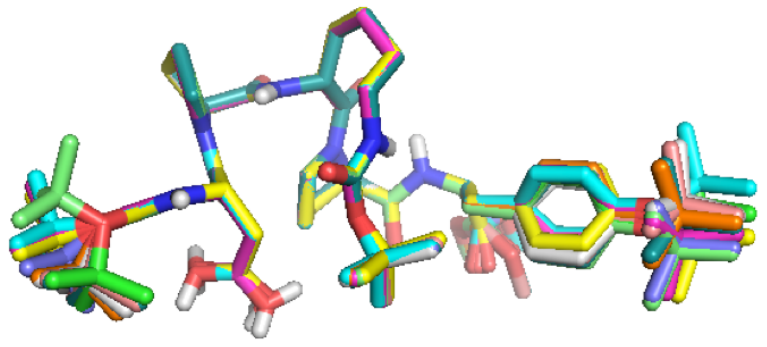

\begin{tabular}{ccc}
\hline Residue & Back & Side \\
\hline Boc & 0.134 & 1.599 \\
Asp & 0.052 & 0.697 \\
Pro & 0.021 & 0.039 \\
Lys & 0.030 & 0.674 \\
DPro & 0.015 & 0.024 \\
Tyr(t-Bu) & 0.027 & 1.327 \\
OMe & 0.000 & 1.315 \\
\hline
\end{tabular}

\begin{tabular}{ccc}
\hline Residue & Back & Side \\
\hline Boc & 0.011 & 1.253 \\
Asp & 0.006 & 0.681 \\
Pro & 0.007 & 0.010 \\
Lys & 0.008 & 0.673 \\
DPro & 0.004 & 0.008 \\
Tyr(t-Bu) & 0.014 & 1.226 \\
OMe & 0.000 & 1.059 \\
\hline
\end{tabular}




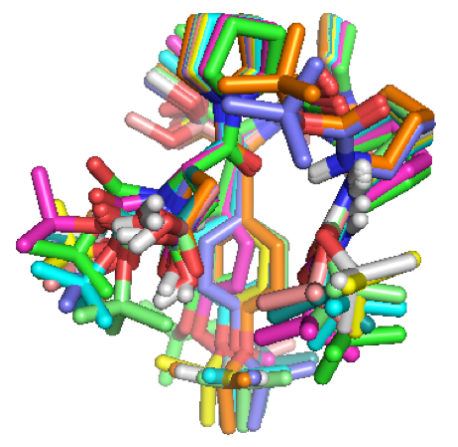

\begin{tabular}{ccc}
\hline Residue & Back & Side \\
\hline Boc & 0.393 & 1.382 \\
Asp & 0.128 & 0.713 \\
Pro & 0.202 & 0.333 \\
Lys & 0.205 & 1.818 \\
DPro & 0.095 & 0.201 \\
Tyr(t-Bu) & 0.197 & 1.438 \\
OMe & 0.000 & 0.946 \\
\hline
\end{tabular}

CNS-Output Coordinates for Peptide 1*

\begin{tabular}{|c|c|c|c|c|c|c|c|c|c|}
\hline Tag & Symbol & $x$ & $Y$ & Z & & & & & \\
\hline 1 & $\mathrm{O}$ & 4.204503 & -0.92079 & -3.536091 & 67 & C & -9.355389 & -0.719963 & -0.37283 \\
\hline 2 & $\mathrm{C}$ & 4.044475 & -1.376427 & -2.411453 & 68 & C & -10.003215 & 0.122646 & 0.730731 \\
\hline 3 & $\mathrm{O}$ & 5.001083 & -1.624568 & -1.494359 & 69 & C & -10.263757 & -0.787465 & -1.603324 \\
\hline 4 & $\mathrm{C}$ & 6.431917 & -1.389267 & -1.788388 & 70 & C & -8.990531 & -2.127942 & 0.108578 \\
\hline 5 & $\mathrm{C}$ & 6.868859 & -2.260833 & -2.969115 & 71 & $\mathrm{H}$ & -9.353295 & 0.190963 & 1.603109 \\
\hline 6 & $\mathrm{C}$ & 6.670784 & 0.102521 & -2.036967 & 72 & $\mathrm{H}$ & -10.952153 & -0.332892 & 1.031014 \\
\hline 7 & $\mathrm{C}$ & 7.116959 & -1.838772 & -0.496155 & 73 & $\mathrm{H}$ & -10.212442 & 1.132432 & 0.363709 \\
\hline 8 & $\mathrm{H}$ & 6.63969 & -3.312428 & -2.770574 & 74 & $\mathrm{H}$ & -10.488636 & 0.217361 & -1.972764 \\
\hline 9 & $\mathrm{H}$ & 7.950639 & -2.169247 & -3.107242 & 75 & $\mathrm{H}$ & -11.205857 & -1.280637 & -1.347312 \\
\hline 10 & $\mathrm{H}$ & 6.370078 & -1.957352 & -3.889677 & 76 & $\mathrm{H}$ & -9.784209 & -1.352776 & -2.407691 \\
\hline 11 & $\mathrm{H}$ & 6.334095 & 0.685425 & -1.174712 & 77 & $\mathrm{H}$ & -8.496734 & -2.68685 & -0.692606 \\
\hline 12 & $\mathrm{H}$ & 6.145816 & 0.441079 & -2.930721 & 78 & $\mathrm{H}$ & -9.900697 & -2.667715 & 0.388641 \\
\hline 13 & $\mathrm{H}$ & 7.742463 & 0.281615 & -2.169735 & 79 & $\mathrm{H}$ & -8.325909 & -2.085352 & 0.971505 \\
\hline 14 & $\mathrm{H}$ & 6.792283 & -1.224839 & 0.348053 & 80 & $\mathrm{~N}$ & 0.319482 & 3.431436 & 1.457692 \\
\hline 15 & $\mathrm{H}$ & 8.201417 & -1.737936 & -0.599222 & 81 & C & 0.94596 & 3.571202 & 2.791585 \\
\hline 16 & $\mathrm{H}$ & 6.887992 & -2.887046 & -0.28285 & 82 & $\mathrm{H}$ & 0.165823 & 3.540357 & 3.555737 \\
\hline 17 & $\mathrm{~N}$ & 2.819951 & -1.739328 & -1.912426 & 83 & C & 1.651001 & 4.952095 & 2.741369 \\
\hline 18 & $\mathrm{H}$ & 2.752215 & -1.903039 & -0.917648 & 84 & $\mathrm{H}$ & 2.70589 & 4.820579 & 2.477913 \\
\hline 19 & $\mathrm{C}$ & 1.619307 & -1.431735 & -2.654729 & 85 & $\mathrm{H}$ & 1.608497 & 5.452433 & 3.71002 \\
\hline 20 & $\mathrm{H}$ & 1.832604 & -1.665411 & -3.698032 & 86 & C & 0.91851 & 5.70655 & 1.624151 \\
\hline 21 & C & 0.468899 & -2.331475 & -2.176631 & 87 & $\mathrm{H}$ & 1.510132 & 6.521635 & 1.199758 \\
\hline 22 & $\mathrm{H}$ & -0.481022 & -1.992031 & -2.605817 & 88 & $\mathrm{H}$ & -0.02452 & 6.123529 & 1.994103 \\
\hline 23 & $\mathrm{H}$ & 0.355027 & -2.281709 & -1.09019 & 89 & C & 0.63082 & 4.602989 & 0.602953 \\
\hline 24 & $\mathrm{C}$ & 0.661405 & -3.771078 & -2.61377 & 90 & $\mathrm{H}$ & 1.520744 & 4.398813 & -0.006053 \\
\hline 25 & $\mathrm{O}$ & 0.097162 & -4.632055 & -1.743626 & 91 & $\mathrm{H}$ & -0.188276 & 4.832784 & -0.074109 \\
\hline 26 & $\mathrm{O}$ & 1.217626 & -4.123995 & -3.631759 & 92 & C & 1.958367 & 2.484909 & 3.16606 \\
\hline 27 & C & 1.218884 & 0.05649 & -2.523557 & 93 & $\mathrm{O}$ & 2.306276 & 2.348016 & 4.336101 \\
\hline 28 & $\mathrm{O}$ & 1.589392 & 0.729293 & -1.548732 & 94 & $\mathrm{~N}$ & 2.487959 & 1.778744 & 2.132929 \\
\hline 29 & $\mathrm{H}$ & 0.218206 & -5.529195 & -2.101672 & 95 & $\mathrm{H}$ & 2.164202 & 1.984782 & 1.198477 \\
\hline 30 & $\mathrm{~N}$ & 0.412803 & 0.570049 & -3.484751 & 96 & C & 3.624864 & 0.898095 & 2.286186 \\
\hline
\end{tabular}




\begin{tabular}{|c|c|c|c|c|c|c|c|c|c|}
\hline 31 & C & -0.017551 & 1.989841 & -3.421834 & 97 & $\mathrm{H}$ & 3.686663 & 0.661074 & 3.354646 \\
\hline 32 & $\mathrm{H}$ & 0.848501 & 2.595376 & -3.139464 & 98 & $C$ & 3.458502 & -0.403063 & 1.468716 \\
\hline 33 & C & -0.519783 & 2.303802 & -4.844865 & 99 & $\mathrm{H}$ & 4.424615 & -0.910866 & 1.46219 \\
\hline 34 & $\mathrm{H}$ & -0.203497 & 3.297551 & -5.168993 & 100 & $\mathrm{H}$ & 3.235446 & -0.121769 & 0.433465 \\
\hline 35 & $\mathrm{H}$ & -1.612162 & 2.273396 & -4.84033 & 101 & $\mathrm{C}$ & 2.38655 & -1.326285 & 2.004439 \\
\hline 36 & C & 0.043387 & 1.17584 & -5.723523 & 102 & $\mathrm{C}$ & 2.703752 & -2.313532 & 2.948115 \\
\hline 37 & $\mathrm{H}$ & 1.070597 & 1.394989 & -6.032237 & 103 & $\mathrm{H}$ & 3.732467 & -2.422475 & 3.282993 \\
\hline 38 & $\mathrm{H}$ & -0.553666 & 1.000537 & -6.621563 & 104 & C & 1.048951 & -1.205484 & 1.600722 \\
\hline 39 & C & 0.039181 & -0.030543 & -4.781244 & 105 & $\mathrm{H}$ & 0.777525 & -0.442578 & 0.877301 \\
\hline 40 & $\mathrm{H}$ & 0.744399 & -0.804987 & -5.08031 & 106 & $\mathrm{C}$ & 1.723472 & -3.154598 & 3.471772 \\
\hline 41 & $\mathrm{H}$ & -0.960579 & -0.477108 & -4.715674 & 107 & $\mathrm{H}$ & 1.976019 & -3.903031 & 4.215371 \\
\hline 42 & C & -1.133504 & 2.153181 & -2.361616 & 108 & C & 0.05917 & -2.041231 & 2.120941 \\
\hline 43 & $\mathrm{O}$ & -2.318297 & 1.957349 & -2.630866 & 109 & $\mathrm{H}$ & -0.977524 & -1.918179 & 1.826858 \\
\hline 44 & $\mathrm{~N}$ & -0.680155 & 2.523486 & -1.133845 & 110 & $\mathrm{C}$ & 0.392404 & -3.028915 & 3.054953 \\
\hline 45 & $\mathrm{H}$ & 0.304736 & 2.316159 & -0.991862 & 111 & $\mathrm{O}$ & -0.590658 & -3.791339 & 3.64621 \\
\hline 46 & $\mathrm{C}$ & -1.552412 & 2.496719 & 0.046945 & 112 & $\mathrm{C}$ & 4.963152 & 1.57299 & 1.936488 \\
\hline 47 & $\mathrm{H}$ & -2.054051 & 1.525287 & 0.091113 & 113 & 0 & 5.993025 & 0.957699 & 1.737119 \\
\hline 48 & C & -2.644727 & 3.601463 & 0.0224 & 114 & C & -0.994974 & -5.07406 & 3.060247 \\
\hline 49 & $\mathrm{H}$ & -3.027949 & 3.660554 & -0.99927 & 115 & C & -2.065699 & -5.569957 & 4.033866 \\
\hline 50 & $\mathrm{H}$ & -2.184543 & 4.569679 & 0.240845 & 116 & $\mathrm{C}$ & -1.587516 & -4.867691 & 1.661772 \\
\hline 51 & C & -3.808223 & 3.342554 & 1.010772 & 117 & $\mathrm{C}$ & 0.191845 & -6.042801 & 3.016091 \\
\hline 52 & $\mathrm{H}$ & -4.086553 & 4.291705 & 1.481336 & 118 & $\mathrm{H}$ & -2.45454 & -6.540848 & 3.713188 \\
\hline 53 & $\mathrm{H}$ & -3.465585 & 2.694238 & 1.826134 & 119 & $\mathrm{H}$ & -2.896465 & -4.860197 & 4.080499 \\
\hline 54 & C & -5.075399 & 2.750284 & 0.370386 & 120 & $\mathrm{H}$ & -1.646922 & -5.676767 & 5.038587 \\
\hline 55 & $\mathrm{H}$ & -5.43209 & 3.426615 & -0.418511 & 121 & $\mathrm{H}$ & -2.407673 & -4.144294 & 1.694897 \\
\hline 56 & $\mathrm{H}$ & -5.867827 & 2.701197 & 1.12608 & 122 & $\mathrm{H}$ & -1.984381 & -5.815646 & 1.284169 \\
\hline 57 & C & -4.895611 & 1.346169 & -0.226503 & 123 & $\mathrm{H}$ & -0.837503 & -4.514708 & 0.948962 \\
\hline 58 & $\mathrm{H}$ & -4.587906 & 0.645684 & 0.555627 & 124 & $\mathrm{H}$ & 0.612388 & -6.185682 & 4.016034 \\
\hline 59 & $\mathrm{H}$ & -4.123763 & 1.352284 & -1.001136 & 125 & $\mathrm{H}$ & 0.982735 & -5.675771 & 2.356005 \\
\hline 60 & $\mathrm{~N}$ & -6.11352 & 0.820966 & -0.831911 & 126 & $\mathrm{H}$ & -0.134807 & -7.017446 & 2.640092 \\
\hline 61 & $\mathrm{H}$ & -6.346794 & 1.087214 & -1.777039 & 127 & 0 & 4.884012 & 2.912302 & 1.911554 \\
\hline 62 & C & -0.732339 & 2.554063 & 1.345453 & 128 & C & 6.124033 & 3.606236 & 1.658574 \\
\hline 63 & $\mathrm{O}$ & -1.036115 & 1.813373 & 2.280016 & 129 & $\mathrm{H}$ & 6.86334 & 3.352395 & 2.420568 \\
\hline 64 & O & -6.991285 & -0.058458 & 1.090935 & 130 & $\mathrm{H}$ & 5.878781 & 4.666194 & 1.700054 \\
\hline 65 & C & -7.089285 & 0.218929 & -0.098681 & 131 & $\mathrm{H}$ & 6.514769 & 3.339949 & 0.674313 \\
\hline 66 & 0 & -8.160334 & -0.038484 & -0.894834 & & & & & \\
\hline
\end{tabular}

*Average of the 10 lowest-energy scored structures generated by CNS.

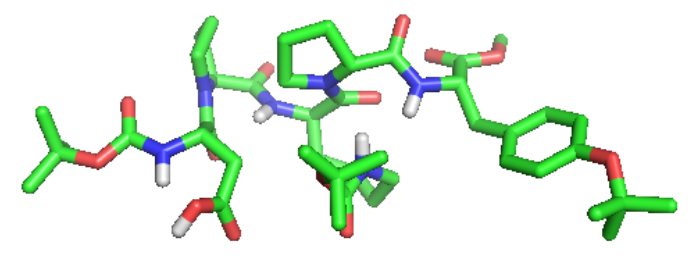




\section{CNS-Output Coordinates for Peptide $1+6^{*}$}

\begin{tabular}{|c|c|c|c|c|c|c|c|c|c|}
\hline Tag & Symbol & $x$ & Y & Z & & & & & \\
\hline 1 & $\mathrm{O}$ & 6.555000 & 2.560000 & 0.516000 & 67 & C & 12.465000 & -0.610000 & -2.356000 \\
\hline 2 & C & 6.324000 & 1.573000 & -0.183000 & 68 & C & 11.445000 & -1.308000 & -3.255000 \\
\hline 3 & $\mathrm{O}$ & 5.182000 & 1.341000 & -0.870000 & 69 & C & 12.614000 & 0.845000 & -2.796000 \\
\hline 4 & C & 4.020000 & 2.228000 & -0.721000 & 70 & C & 13.809000 & -1.321000 & -2.438000 \\
\hline 5 & C & 2.861000 & 1.695000 & -1.561000 & 71 & $\mathrm{H}$ & 11.387000 & -2.351000 & -2.986000 \\
\hline 6 & C & 3.598000 & 2.280000 & 0.747000 & 72 & $\mathrm{H}$ & 11.754000 & -1.217000 & -4.284000 \\
\hline 7 & C & 4.419000 & 3.617000 & -1.201000 & 73 & $\mathrm{H}$ & 10.473000 & -0.851000 & -3.130000 \\
\hline 8 & $\mathrm{H}$ & 3.253000 & 1.130000 & -2.392000 & 74 & $\mathrm{H}$ & 11.652000 & 1.225000 & -3.104000 \\
\hline 9 & $\mathrm{H}$ & 2.277000 & 2.524000 & -1.930000 & 75 & $\mathrm{H}$ & 13.306000 & 0.899000 & -3.622000 \\
\hline 10 & $\mathrm{H}$ & 2.233000 & 1.055000 & -0.957000 & 76 & $\mathrm{H}$ & 12.988000 & 1.440000 & -1.974000 \\
\hline 11 & $\mathrm{H}$ & 3.087000 & 3.210000 & 0.939000 & 77 & $\mathrm{H}$ & 14.601000 & -0.603000 & -2.298000 \\
\hline 12 & $\mathrm{H}$ & 4.474000 & 2.213000 & 1.373000 & 78 & $\mathrm{H}$ & 13.909000 & -1.785000 & -3.406000 \\
\hline 13 & $\mathrm{H}$ & 2.936000 & 1.454000 & 0.968000 & 79 & $\mathrm{H}$ & 13.871000 & -2.079000 & -1.669000 \\
\hline 14 & $\mathrm{H}$ & 5.478000 & 3.756000 & -1.049000 & 80 & $N$ & 12.574000 & 1.256000 & 2.521000 \\
\hline 15 & $\mathrm{H}$ & 3.872000 & 4.358000 & -0.641000 & 81 & C & 13.413000 & 2.424000 & 2.336000 \\
\hline 16 & $\mathrm{H}$ & 4.191000 & 3.721000 & -2.253000 & 82 & $\mathrm{H}$ & 13.736000 & 2.853000 & 3.269000 \\
\hline 17 & $\mathrm{~N}$ & 7.187000 & 0.572000 & -0.362000 & 83 & C & 12.406000 & 3.329000 & 1.638000 \\
\hline 18 & $\mathrm{H}$ & 6.956000 & -0.130000 & -0.999000 & 84 & $\mathrm{H}$ & 12.891000 & 4.185000 & 1.201000 \\
\hline 19 & C & 8.573000 & 0.655000 & 0.066000 & 85 & $\mathrm{H}$ & 11.620000 & 3.633000 & 2.348000 \\
\hline 20 & $\mathrm{H}$ & 8.907000 & -0.335000 & 0.354000 & 86 & C & 11.863000 & 2.405000 & 0.617000 \\
\hline 21 & C & 9.466000 & 1.074000 & -1.084000 & 87 & $\mathrm{H}$ & 12.605000 & 2.203000 & -0.140000 \\
\hline 22 & $\mathrm{H}$ & 9.169000 & 0.518000 & -1.957000 & 88 & $\mathrm{H}$ & 10.971000 & 2.816000 & 0.180000 \\
\hline 23 & $\mathrm{H}$ & 10.486000 & 0.806000 & -0.831000 & 89 & C & 11.567000 & 1.157000 & 1.414000 \\
\hline 24 & C & 9.391000 & 2.556000 & -1.404000 & 90 & $\mathrm{H}$ & 11.691000 & 0.266000 & 0.821000 \\
\hline 25 & $\mathrm{O}$ & 10.368000 & 3.092000 & -1.967000 & 91 & $\mathrm{H}$ & 10.565000 & 1.190000 & 1.796000 \\
\hline 26 & $\mathrm{O}$ & 8.355000 & 3.179000 & -1.091000 & 92 & C & 14.609000 & 2.108000 & 1.455000 \\
\hline 27 & C & 8.794000 & 1.501000 & 1.296000 & 93 & $\mathrm{O}$ & 14.889000 & 2.810000 & 0.483000 \\
\hline 28 & $\mathrm{O}$ & 9.439000 & 2.544000 & 1.261000 & 94 & $N$ & 15.405000 & 1.159000 & 1.926000 \\
\hline 29 & $\mathrm{H}$ & 10.016000 & 3.517000 & -2.765000 & 95 & $\mathrm{H}$ & 15.177000 & 0.742000 & 2.777000 \\
\hline 30 & $N$ & 8.347000 & 0.981000 & 2.436000 & 96 & C & 16.585000 & 0.696000 & 1.208000 \\
\hline 31 & C & 8.631000 & 1.586000 & 3.713000 & 97 & $\mathrm{H}$ & 17.207000 & 0.157000 & 1.905000 \\
\hline 32 & $\mathrm{H}$ & 8.620000 & 2.673000 & 3.677000 & 98 & C & 16.151000 & -0.257000 & 0.093000 \\
\hline 33 & C & 7.562000 & 0.996000 & 4.620000 & 99 & $\mathrm{H}$ & 15.759000 & 0.327000 & -0.723000 \\
\hline 34 & $\mathrm{H}$ & 6.656000 & 1.578000 & 4.548000 & 100 & $\mathrm{H}$ & 15.368000 & -0.906000 & 0.468000 \\
\hline 35 & $\mathrm{H}$ & 7.915000 & 0.981000 & 5.641000 & 101 & C & 17.263000 & -1.136000 & -0.449000 \\
\hline 36 & C & 7.371000 & -0.387000 & 4.076000 & 102 & C & 16.980000 & -2.161000 & -1.344000 \\
\hline 37 & $\mathrm{H}$ & 6.360000 & -0.718000 & 4.263000 & 103 & $\mathrm{H}$ & 15.957000 & -2.324000 & -1.649000 \\
\hline 38 & $\mathrm{H}$ & 8.077000 & -1.062000 & 4.537000 & 104 & C & 18.589000 & -0.945000 & -0.071000 \\
\hline 39 & C & 7.627000 & -0.299000 & 2.584000 & 105 & $\mathrm{H}$ & 18.828000 & -0.154000 & 0.624000 \\
\hline 40 & $\mathrm{H}$ & 6.690000 & -0.273000 & 2.047000 & 106 & C & 17.983000 & -2.970000 & -1.844000 \\
\hline
\end{tabular}




\begin{tabular}{|c|c|c|c|c|c|c|c|c|c|}
\hline 41 & $\mathrm{H}$ & 8.233000 & -1.125000 & 2.240000 & 107 & $\mathrm{H}$ & 17.742000 & -3.761000 & -2.539000 \\
\hline 42 & C & 10.024000 & 1.096000 & 4.074000 & 108 & $\mathrm{C}$ & 19.596000 & -1.749000 & -0.568000 \\
\hline 43 & 0 & 10.729000 & 1.683000 & 4.895000 & 109 & $\mathrm{H}$ & 20.619000 & -1.585000 & -0.262000 \\
\hline 44 & $\mathrm{~N}$ & 10.353000 & -0.076000 & 3.487000 & 110 & $C$ & 19.288000 & -2.760000 & -1.454000 \\
\hline 45 & $\mathrm{H}$ & 9.705000 & -0.502000 & 2.904000 & 111 & 0 & 20.290000 & -3.563000 & -1.951000 \\
\hline 46 & C & 11.664000 & -0.672000 & 3.570000 & 112 & $\mathrm{C}$ & 17.387000 & 1.861000 & 0.630000 \\
\hline 47 & $\mathrm{H}$ & 11.788000 & -1.214000 & 2.654000 & 113 & 0 & 17.437000 & 2.075000 & -0.582000 \\
\hline 48 & C & 11.846000 & -1.638000 & 4.732000 & 114 & $C$ & 21.044000 & -3.166000 & -3.147000 \\
\hline 49 & $\mathrm{H}$ & 10.897000 & -1.782000 & 5.226000 & 115 & C & 21.708000 & -1.810000 & -2.909000 \\
\hline 50 & $\mathrm{H}$ & 12.553000 & -1.218000 & 5.434000 & 116 & $C$ & 22.120000 & -4.210000 & -3.442000 \\
\hline 51 & C & 12.364000 & -3.005000 & 4.273000 & 117 & $\mathrm{C}$ & 20.069000 & -3.075000 & -4.313000 \\
\hline 52 & $\mathrm{H}$ & 11.519000 & -3.660000 & 4.112000 & 118 & $\mathrm{H}$ & 20.947000 & -1.069000 & -2.719000 \\
\hline 53 & $\mathrm{H}$ & 12.993000 & -3.417000 & 5.048000 & 119 & $\mathrm{H}$ & 22.274000 & -1.531000 & -3.783000 \\
\hline 54 & C & 13.180000 & -2.916000 & 2.971000 & 120 & $\mathrm{H}$ & 22.370000 & -1.870000 & -2.056000 \\
\hline 55 & $\mathrm{H}$ & 13.917000 & -3.706000 & 2.969000 & 121 & $\mathrm{H}$ & 22.620000 & -3.957000 & -4.364000 \\
\hline 56 & $\mathrm{H}$ & 13.679000 & -1.960000 & 2.937000 & 122 & $\mathrm{H}$ & 21.658000 & -5.180000 & -3.536000 \\
\hline 57 & C & 12.297000 & -3.056000 & 1.723000 & 123 & $\mathrm{H}$ & 22.841000 & -4.232000 & -2.636000 \\
\hline 58 & $\mathrm{H}$ & 11.262000 & -3.052000 & 2.033000 & 124 & $\mathrm{H}$ & 20.623000 & -3.015000 & -5.237000 \\
\hline 59 & $\mathrm{H}$ & 12.522000 & -4.000000 & 1.249000 & 125 & $\mathrm{H}$ & 19.459000 & -2.193000 & -4.199000 \\
\hline 60 & $\mathrm{~N}$ & 12.505000 & -1.973000 & 0.740000 & 126 & $\mathrm{H}$ & 19.436000 & -3.951000 & -4.331000 \\
\hline 61 & $\mathrm{H}$ & 13.060000 & -1.215000 & 1.020000 & 127 & 0 & 18.088000 & 2.502000 & 1.591000 \\
\hline 62 & C & 12.678000 & 0.429000 & 3.566000 & 128 & $C$ & 18.926000 & 3.558000 & 1.149000 \\
\hline 63 & $\mathrm{O}$ & 13.495000 & 0.577000 & 4.474000 & 129 & $\mathrm{H}$ & 19.478000 & 3.959000 & 1.984000 \\
\hline 64 & 0 & 10.966000 & -2.697000 & -0.741000 & 130 & $\mathrm{H}$ & 18.327000 & 4.341000 & 0.714000 \\
\hline 65 & C & 11.757000 & -1.838000 & -0.353000 & 131 & $\mathrm{H}$ & 19.628000 & 3.203000 & 0.406000 \\
\hline 66 & $\mathrm{O}$ & 12.008000 & -0.656000 & -0.960000 & & & & & \\
\hline
\end{tabular}

*Average of the 10 lowest-energy scored structures generated by CNS.

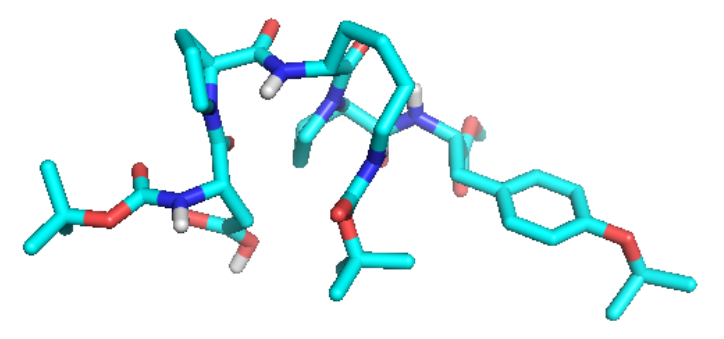

\section{CNS-Output Coordinates for Peptide $1+\mathbf{8}^{*}$}

$\begin{array}{cccccccccc}\text { Tag } & \text { Symbol } & X & Y & Z & & & & & \\ 1 & \mathrm{O} & 6.587000 & 2.525000 & 0.492000 & 67 & \mathrm{C} & 12.586000 & -0.293000 & -2.504000 \\ 2 & \mathrm{C} & 6.379000 & 1.513000 & -0.176000 & 68 & \mathrm{C} & 11.440000 & -0.723000 & -3.419000 \\ 3 & \mathrm{O} & 5.256000 & 1.244000 & -0.880000 & 69 & \mathrm{C} & 13.032000 & 1.118000 & -2.882000 \\ 4 & \mathrm{C} & 4.332000 & 2.323000 & -1.260000 & 70 & \mathrm{C} & 13.752000 & -1.263000 & -2.632000\end{array}$




\begin{tabular}{|c|c|c|c|c|c|c|c|c|c|}
\hline 5 & $\mathrm{C}$ & 5.077000 & 3.367000 & -2.092000 & 71 & $\mathrm{H}$ & 11.765000 & -0.670000 & -4.447000 \\
\hline 6 & C & 3.186000 & 1.743000 & -2.087000 & 72 & $\mathrm{H}$ & 10.599000 & -0.064000 & -3.272000 \\
\hline 7 & C & 3.791000 & 2.955000 & 0.014000 & 73 & $\mathrm{H}$ & 11.146000 & -1.738000 & -3.188000 \\
\hline 8 & 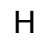 & 5.895000 & 2.893000 & -2.610000 & 74 & $\mathrm{H}$ & 13.515000 & 1.579000 & -2.034000 \\
\hline 9 & $\mathrm{H}$ & 5.459000 & 4.136000 & -1.439000 & 75 & $\mathrm{H}$ & 12.170000 & 1.700000 & -3.168000 \\
\hline 10 & $\mathrm{H}$ & 4.403000 & 3.810000 & -2.813000 & 76 & $\mathrm{H}$ & 13.726000 & 1.074000 & -3.710000 \\
\hline 11 & $\mathrm{H}$ & 3.550000 & 1.481000 & -3.068000 & 77 & $\mathrm{H}$ & 14.644000 & -0.801000 & -2.240000 \\
\hline 12 & $\mathrm{H}$ & 2.403000 & 2.479000 & -2.176000 & 78 & $\mathrm{H}$ & 13.899000 & -1.508000 & -3.672000 \\
\hline 13 & $\mathrm{H}$ & 2.794000 & 0.859000 & -1.602000 & 79 & $\mathrm{H}$ & 13.541000 & -2.167000 & -2.077000 \\
\hline 14 & $\mathrm{H}$ & 3.468000 & 2.177000 & 0.688000 & 80 & $\mathrm{~N}$ & 12.608000 & 1.397000 & 2.602000 \\
\hline 15 & $\mathrm{H}$ & 2.956000 & 3.592000 & -0.231000 & 81 & C & 13.423000 & 2.586000 & 2.477000 \\
\hline 16 & $\mathrm{H}$ & 4.564000 & 3.543000 & 0.489000 & 82 & $\mathrm{H}$ & 13.765000 & 2.956000 & 3.426000 \\
\hline 17 & $\mathrm{~N}$ & 7.256000 & 0.514000 & -0.301000 & 83 & $\mathrm{C}$ & 12.387000 & 3.515000 & 1.857000 \\
\hline 18 & $\mathrm{H}$ & 7.046000 & -0.212000 & -0.917000 & 84 & $\mathrm{H}$ & 12.849000 & 4.409000 & 1.472000 \\
\hline 19 & C & 8.630000 & 0.630000 & 0.156000 & 85 & $\mathrm{H}$ & 11.608000 & 3.755000 & 2.597000 \\
\hline 20 & $\mathrm{H}$ & 8.979000 & -0.347000 & 0.461000 & 86 & C & 11.845000 & 2.652000 & 0.783000 \\
\hline 21 & C & 9.545000 & 1.047000 & -0.979000 & 87 & $\mathrm{H}$ & 12.573000 & 2.528000 & -0.003000 \\
\hline 22 & $\mathrm{H}$ & 9.246000 & 0.511000 & -1.864000 & 88 & $\mathrm{H}$ & 10.932000 & 3.070000 & 0.399000 \\
\hline 23 & $\mathrm{H}$ & 10.557000 & 0.751000 & -0.718000 & 89 & C & 11.599000 & 1.337000 & 1.492000 \\
\hline 24 & $C$ & 9.510000 & 2.535000 & -1.279000 & 90 & $\mathrm{H}$ & 11.753000 & 0.493000 & 0.837000 \\
\hline 25 & $\mathrm{O}$ & 10.513000 & 3.058000 & -1.807000 & 91 & $\mathrm{H}$ & 10.597000 & 1.304000 & 1.876000 \\
\hline 26 & $\mathrm{O}$ & 8.478000 & 3.175000 & -0.986000 & 92 & $C$ & 14.595000 & 2.331000 & 1.548000 \\
\hline 27 & $C$ & 8.809000 & 1.482000 & 1.385000 & 93 & 0 & 14.895000 & 3.128000 & 0.659000 \\
\hline 28 & $\mathrm{O}$ & 9.417000 & 2.546000 & 1.358000 & 94 & $\mathrm{~N}$ & 15.348000 & 1.301000 & 1.898000 \\
\hline 29 & $\mathrm{H}$ & 10.421000 & 4.014000 & -1.667000 & 95 & $\mathrm{H}$ & 15.104000 & 0.802000 & 2.701000 \\
\hline 30 & $\mathrm{~N}$ & 8.379000 & 0.939000 & 2.520000 & 96 & $C$ & 16.499000 & 0.866000 & 1.119000 \\
\hline 31 & C & 8.641000 & 1.543000 & 3.800000 & 97 & $\mathrm{H}$ & 17.106000 & 0.234000 & 1.749000 \\
\hline 32 & $\mathrm{H}$ & 8.607000 & 2.631000 & 3.774000 & 98 & $C$ & 16.012000 & 0.050000 & -0.078000 \\
\hline 33 & $C$ & 7.586000 & 0.918000 & 4.699000 & 99 & $\mathrm{H}$ & 15.614000 & 0.725000 & -0.818000 \\
\hline 34 & $\mathrm{H}$ & 6.663000 & 1.473000 & 4.625000 & 100 & $\mathrm{H}$ & 15.224000 & -0.624000 & 0.246000 \\
\hline 35 & $\mathrm{H}$ & 7.934000 & 0.911000 & 5.723000 & 101 & C & 17.095000 & -0.778000 & -0.733000 \\
\hline 36 & $C$ & 7.439000 & -0.468000 & 4.151000 & 102 & $\mathrm{C}$ & 17.941000 & -0.223000 & -1.684000 \\
\hline 37 & $\mathrm{H}$ & 6.435000 & -0.826000 & 4.324000 & 103 & $\mathrm{H}$ & 17.816000 & 0.815000 & -1.955000 \\
\hline 38 & $\mathrm{H}$ & 8.157000 & -1.125000 & 4.619000 & 104 & $\mathrm{C}$ & 17.272000 & -2.115000 & -0.399000 \\
\hline 39 & C & 7.710000 & -0.371000 & 2.661000 & 105 & $\mathrm{H}$ & 16.622000 & -2.562000 & 0.339000 \\
\hline 40 & $\mathrm{H}$ & 6.780000 & -0.384000 & 2.112000 & 106 & $C$ & 18.931000 & -0.976000 & -2.285000 \\
\hline 41 & $\mathrm{H}$ & 8.354000 & -1.171000 & 2.326000 & 107 & $\mathrm{H}$ & 19.579000 & -0.525000 & -3.023000 \\
\hline 42 & $C$ & 10.045000 & 1.074000 & 4.150000 & 108 & $C$ & 18.260000 & -2.875000 & -0.994000 \\
\hline 43 & $\mathrm{O}$ & 10.714000 & 1.607000 & 5.036000 & 109 & $\mathrm{H}$ & 18.380000 & -3.912000 & -0.720000 \\
\hline 44 & $\mathrm{~N}$ & 10.420000 & -0.031000 & 3.466000 & 110 & $C$ & 19.088000 & -2.301000 & -1.937000 \\
\hline 45 & $\mathrm{H}$ & 9.795000 & -0.419000 & 2.835000 & 111 & $\mathrm{O}$ & 20.079000 & -3.052000 & -2.537000 \\
\hline 46 & C & 11.741000 & -0.602000 & 3.518000 & 112 & $C$ & 17.344000 & 2.049000 & 0.648000 \\
\hline 47 & $\mathrm{H}$ & 11.885000 & -1.065000 & 2.563000 & 113 & $\mathrm{O}$ & 17.109000 & 2.632000 & -0.411000 \\
\hline
\end{tabular}




$\begin{array}{llllllllll}48 & \mathrm{C} & 11.924000 & -1.660000 & 4.596000 & 114 & \mathrm{C} & 19.849000 & -4.453000 & -2.935000 \\ 49 & \mathrm{H} & 11.033000 & -1.706000 & 5.203000 & 115 & \mathrm{C} & 18.408000 & -4.634000 & -3.417000 \\ 50 & \mathrm{H} & 12.767000 & -1.391000 & 5.216000 & 116 & \mathrm{C} & 20.806000 & -4.819000 & -4.069000 \\ 51 & \mathrm{C} & 12.176000 & -3.049000 & 3.999000 & 117 & \mathrm{C} & 20.114000 & -5.337000 & -1.721000 \\ 52 & \mathrm{H} & 11.225000 & -3.540000 & 3.848000 & 118 & \mathrm{H} & 17.777000 & -4.871000 & -2.574000 \\ 53 & \mathrm{H} & 12.769000 & -3.624000 & 4.694000 & 119 & \mathrm{H} & 18.370000 & -5.438000 & -4.135000 \\ 54 & \mathrm{C} & 12.920000 & -2.982000 & 2.653000 & 120 & \mathrm{H} & 18.059000 & -3.722000 & -3.881000 \\ 55 & \mathrm{H} & 13.498000 & -3.887000 & 2.530000 & 121 & \mathrm{H} & 20.361000 & -4.545000 & -5.013000 \\ 56 & \mathrm{H} & 13.585000 & -2.131000 & 2.665000 & 122 & \mathrm{H} & 20.990000 & -5.882000 & -4.051000 \\ 57 & \mathrm{C} & 11.961000 & -2.840000 & 1.460000 & 123 & \mathrm{H} & 21.741000 & -4.290000 & -3.945000 \\ 58 & \mathrm{H} & 10.974000 & -2.615000 & 1.837000 & 124 & \mathrm{H} & 21.042000 & -5.869000 & -1.863000 \\ 59 & \mathrm{H} & 11.933000 & -3.779000 & 0.929000 & 125 & \mathrm{H} & 19.306000 & -6.043000 & -1.609000 \\ 60 & \mathrm{~N} & 12.366000 & -1.775000 & 0.517000 & 126 & \mathrm{H} & 20.184000 & -4.727000 & -0.831000 \\ 61 & \mathrm{H} & 13.073000 & -1.163000 & 0.812000 & 127 & \mathrm{O} & 18.399000 & 2.258000 & 1.467000 \\ 62 & \mathrm{C} & 12.732000 & 0.511000 & 3.595000 & 128 & \mathrm{C} & 19.292000 & 3.291000 & 1.078000 \\ 63 & \mathrm{O} & 13.550000 & 0.613000 & 4.509000 & 129 & \mathrm{H} & 18.865000 & 4.251000 & 1.319000 \\ 64 & \mathrm{O} & 10.687000 & -2.092000 & -0.954000 & 130 & \mathrm{H} & 19.469000 & 3.239000 & 0.017000 \\ 65 & \mathrm{C} & 11.646000 & -1.436000 & -0.546000 & 131 & \mathrm{H} & 20.238000 & 3.191000 & 1.595000 \\ 66 & \mathrm{O} & 12.133000 & -0.305000 & -1.106000 & & & & & \end{array}$

*Average of the 10 lowest-energy scored structures generated by CNS.

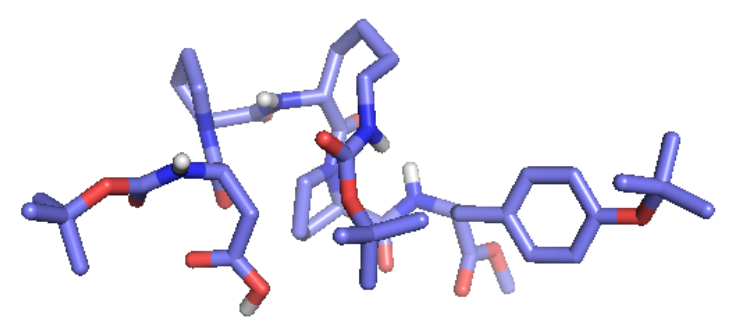

\section{CNS-Output Coordinates for ent-1 + 6*}

$\begin{array}{cccccccccc}\text { Tag } & \text { Symbol } & \mathrm{X} & \mathrm{Y} & \mathrm{Z} & & & & & \\ 1 & \mathrm{O} & 6.745827 & -1.960126 & -1.028094 & 67 & \mathrm{C} & -0.809455 & -2.86035 & 2.733599 \\ 2 & \mathrm{C} & 7.170903 & -1.313084 & -0.076542 & 68 & \mathrm{C} & 0.465643 & -2.912699 & 3.582256 \\ 3 & \mathrm{O} & 8.465644 & -1.125615 & 0.251279 & 69 & \mathrm{C} & -2.014515 & -3.470712 & 3.456714 \\ 4 & \mathrm{C} & 9.558786 & -1.712265 & -0.549958 & 70 & \mathrm{C} & -0.582207 & -3.550845 & 1.386182 \\ 5 & \mathrm{C} & 9.462161 & -3.240312 & -0.524987 & 71 & \mathrm{H} & 1.294456 & -2.434228 & 3.050545 \\ 6 & \mathrm{C} & 9.525509 & -1.145469 & -1.972257 & 72 & \mathrm{H} & 0.739508 & -3.955807 & 3.769613 \\ 7 & \mathrm{C} & 10.806433 & -1.234753 & 0.196127 & 73 & \mathrm{H} & 0.322056 & -2.408135 & 4.53724 \\ 8 & \mathrm{H} & 8.564973 & -3.586091 & -1.038873 & 74 & \mathrm{H} & -2.911171 & -3.393666 & 2.833696 \\ 9 & \mathrm{H} & 9.443396 & -3.602653 & 0.507469 & 75 & \mathrm{H} & -2.198226 & -2.96347 & 4.403903 \\ 10 & \mathrm{H} & 10.339146 & -3.668943 & -1.019837 & 76 & \mathrm{H} & -1.826704 & -4.531 & 3.653951 \\ 11 & \mathrm{H} & 10.404539 & -1.492851 & -2.524026 & 77 & \mathrm{H} & -0.3459 & -4.60745 & 1.541412\end{array}$




\begin{tabular}{|c|c|c|c|c|c|c|c|c|c|}
\hline 12 & $\mathrm{H}$ & 9.551207 & -0.051751 & -1.945807 & 78 & $\mathrm{H}$ & 0.248875 & -3.085529 & 0.848008 \\
\hline 13 & $\mathrm{H}$ & 8.62907 & -1.466683 & -2.50305 & 79 & $\mathrm{H}$ & -1.476904 & -3.484445 & 0.760402 \\
\hline 14 & $\mathrm{H}$ & 10.849032 & -0.142125 & 0.219336 & 80 & $\mathrm{~N}$ & -1.447121 & 2.72275 & -1.970578 \\
\hline 15 & $\mathrm{H}$ & 11.704938 & -1.605972 & -0.304779 & 81 & $C$ & -2.641947 & 2.104889 & -2.608299 \\
\hline 16 & $\mathrm{H}$ & 10.80619 & -1.603972 & 1.225688 & 82 & $\mathrm{H}$ & -2.482517 & 1.02266 & -2.624176 \\
\hline 17 & $N$ & 6.371997 & -0.689056 & 0.840237 & 83 & $C$ & -2.693664 & 2.721807 & -4.01923 \\
\hline 18 & $\mathrm{H}$ & 6.79631 & -0.035181 & 1.484799 & 84 & $\mathrm{H}$ & -3.499811 & 3.458956 & -4.03939 \\
\hline 19 & C & 4.936782 & -0.606691 & 0.639906 & 85 & $\mathrm{H}$ & -2.909156 & 1.96463 & -4.776553 \\
\hline 20 & $\mathrm{H}$ & 4.633062 & -1.540793 & 0.168551 & 86 & C & -1.328181 & 3.398909 & -4.216566 \\
\hline 21 & C & 4.186891 & -0.494095 & 1.987206 & 87 & $\mathrm{H}$ & -1.369977 & 4.24074 & -4.912066 \\
\hline 22 & $\mathrm{H}$ & 4.487264 & -1.340438 & 2.613885 & 88 & $\mathrm{H}$ & -0.585692 & 2.686174 & -4.591633 \\
\hline 23 & $\mathrm{H}$ & 3.111556 & -0.56148 & 1.811712 & 89 & $\mathrm{C}$ & -0.941366 & 3.837956 & -2.80195 \\
\hline 24 & C & 4.425998 & 0.784576 & 2.769813 & 90 & $\mathrm{H}$ & -1.457822 & 4.763517 & -2.520199 \\
\hline 25 & 0 & 5.725592 & 0.924779 & 3.129314 & 91 & $\mathrm{H}$ & 0.132795 & 3.969534 & -2.680819 \\
\hline 26 & 0 & 3.568608 & 1.572549 & 3.102338 & 92 & $\mathrm{C}$ & -3.939424 & 2.3889 & -1.805258 \\
\hline 27 & C & 4.58566 & 0.605575 & -0.24883 & 93 & 0 & -4.773384 & 3.221147 & -2.159812 \\
\hline 28 & $\mathrm{O}$ & 4.915707 & 1.735876 & 0.115269 & 94 & $\mathrm{~N}$ & -4.092572 & 1.614177 & -0.704871 \\
\hline 29 & $\mathrm{H}$ & 5.803882 & 1.771068 & 3.603047 & 95 & $\mathrm{H}$ & -3.275878 & 1.091755 & -0.396228 \\
\hline 30 & $\mathrm{~N}$ & 3.88511 & 0.392443 & -1.398423 & 96 & $\mathrm{C}$ & -5.234124 & 1.77741 & 0.167858 \\
\hline 31 & C & 3.662265 & 1.527291 & -2.31127 & 97 & $\mathrm{H}$ & -6.13683 & 1.827756 & -0.447666 \\
\hline 32 & $\mathrm{H}$ & 4.525854 & 2.195194 & -2.285762 & 98 & $C$ & -5.35246 & 0.584717 & 1.151115 \\
\hline 33 & C & 3.486127 & 0.853152 & -3.68808 & 99 & $\mathrm{H}$ & -6.191211 & 0.79918 & 1.821355 \\
\hline 34 & $\mathrm{H}$ & 4.467975 & 0.738735 & -4.157634 & 100 & $\mathrm{H}$ & -4.448593 & 0.548713 & 1.770084 \\
\hline 35 & $\mathrm{H}$ & 2.860322 & 1.449279 & -4.354853 & 101 & $C$ & -5.556248 & -0.745118 & 0.458516 \\
\hline 36 & C & 2.894261 & -0.522427 & -3.344751 & 102 & $C$ & -6.787634 & -1.073086 & -0.131133 \\
\hline 37 & $\mathrm{H}$ & 3.042582 & -1.26203 & -4.134663 & 103 & $\mathrm{H}$ & -7.61634 & -0.371005 & -0.076252 \\
\hline 38 & $\mathrm{H}$ & 1.817909 & -0.438121 & -3.160933 & 104 & $C$ & -4.527422 & -1.685116 & 0.370614 \\
\hline 39 & C & 3.621554 & -0.916358 & -2.051592 & 105 & $\mathrm{H}$ & -3.562712 & -1.471306 & 0.824583 \\
\hline 40 & $\mathrm{H}$ & 4.568443 & -1.428018 & -2.248712 & 106 & $C$ & -6.973929 & -2.284371 & -0.782825 \\
\hline 41 & $\mathrm{H}$ & 3.008008 & -1.556847 & -1.412014 & 107 & $\mathrm{H}$ & -7.928688 & -2.534577 & -1.234128 \\
\hline 42 & C & 2.462556 & 2.416215 & -1.939681 & 108 & $C$ & -4.698717 & -2.911301 & -0.281683 \\
\hline 43 & $\mathrm{O}$ & 2.321494 & 3.506323 & -2.496265 & 109 & $\mathrm{H}$ & -3.866145 & -3.598901 & -0.317162 \\
\hline 44 & $\mathrm{~N}$ & 1.600109 & 1.914977 & -1.022877 & 110 & C & -5.930585 & -3.224695 & -0.871184 \\
\hline 45 & $\mathrm{H}$ & 1.843251 & 1.03438 & -0.589818 & 111 & 0 & -6.267361 & -4.363612 & -1.543274 \\
\hline 46 & C & 0.510041 & 2.679417 & -0.4145 & 112 & $C$ & -5.155645 & 3.093569 & 0.950621 \\
\hline 47 & $\mathrm{H}$ & 0.643857 & 3.717784 & -0.719151 & 113 & 0 & -4.137357 & 3.701337 & 1.211556 \\
\hline 48 & C & 0.588698 & 2.557714 & 1.112251 & 114 & $C$ & -5.403436 & -5.515879 & -1.77889 \\
\hline 49 & $\mathrm{H}$ & 1.602407 & 2.82714 & 1.424769 & 115 & $\mathrm{C}$ & -4.199999 & -5.136748 & -2.654134 \\
\hline 50 & $\mathrm{H}$ & 0.455631 & 1.503636 & 1.372159 & 116 & $C$ & -4.999266 & -6.18448 & -0.457548 \\
\hline 51 & C & -0.440471 & 3.42428 & 1.860397 & 117 & C & -6.328946 & -6.454417 & -2.564101 \\
\hline 52 & $\mathrm{H}$ & -1.39059 & 3.46233 & 1.312246 & 118 & $\mathrm{H}$ & -4.542986 & -4.631111 & -3.561496 \\
\hline 53 & $\mathrm{H}$ & -0.070465 & 4.455642 & 1.891133 & 119 & $\mathrm{H}$ & -3.664419 & -6.04372 & -2.950876 \\
\hline 54 & C & -0.7218 & 2.927847 & 3.293716 & 120 & $\mathrm{H}$ & -3.488934 & -4.482847 & -2.147036 \\
\hline
\end{tabular}




$\begin{array}{llllllllll}55 & \mathrm{H} & -1.015855 & 3.777799 & 3.921478 & 121 & \mathrm{H} & -5.890376 & -6.407209 & 0.136439 \\ 56 & \mathrm{H} & 0.193268 & 2.513998 & 3.736211 & 122 & \mathrm{H} & -4.331342 & -5.569653 & 0.147466 \\ 57 & \mathrm{C} & -1.861688 & 1.900175 & 3.389443 & 123 & \mathrm{H} & -4.484963 & -7.127146 & -0.668119 \\ 58 & \mathrm{H} & -2.7826 & 2.364631 & 3.019497 & 124 & \mathrm{H} & -7.211801 & -6.70485 & -1.969413 \\ 59 & \mathrm{H} & -2.022541 & 1.614923 & 4.430867 & 125 & \mathrm{H} & -5.805837 & -7.380521 & -2.818313 \\ 60 & \mathrm{~N} & -1.642233 & 0.659789 & 2.650284 & 126 & \mathrm{H} & -6.66054 & -5.976012 & -3.48988 \\ 61 & \mathrm{H} & -1.537078 & 0.692475 & 1.639886 & 127 & \mathrm{O} & -6.380154 & 3.470514 & 1.355114 \\ 62 & \mathrm{C} & -0.833981 & 2.117653 & -0.933623 & 128 & \mathrm{C} & -6.439858 & 4.670665 & 2.152986 \\ 63 & \mathrm{O} & -1.308316 & 1.091713 & -0.401996 & 129 & \mathrm{H} & -7.493393 & 4.814193 & 2.387744 \\ 64 & \mathrm{O} & -1.367664 & -0.689228 & 4.488217 & 130 & \mathrm{H} & -6.053707 & 5.520998 & 1.587406 \\ 65 & \mathrm{C} & -1.365538 & -0.515991 & 3.274882 & 131 & \mathrm{H} & -5.855607 & 4.549989 & 3.067729 \\ 66 & \mathrm{O} & -1.102646 & -1.473852 & 2.339932 & & & & & \end{array}$

*Average of the 10 lowest-energy scored structures generated by CNS.

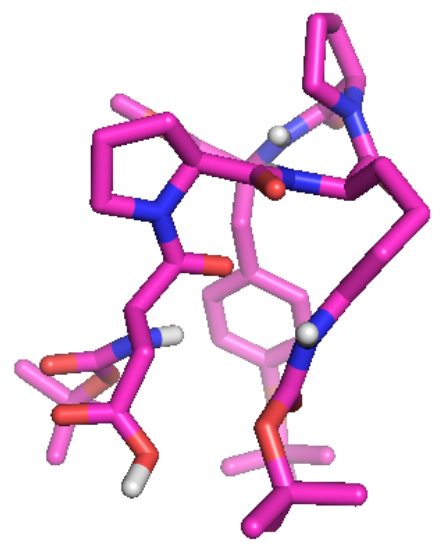

\section{References}

1. Fulmer, G. R.; Miller, A. J. M.; Sherden, N. H.; Gottlieb, H. E.; Nudelman, A.; Stoltz, B. M.; Bercaw, J. E.; Goldberg, K. I. Organometallics, 2010, 29, 2176.

2. Romney, D. K.; Colvin, S. M.; Miller, S. J. J. Am. Chem. Soc. 2014, 136, 14019.

3. Borgia, B. A.; Gochin, M.; Kehrwood, D. J.; James, T. L. Prog. NMR Spectrosc., 1990, 22, 83.

4. Macura, S.; Farmer II, B. T.; Brown, L. R. J. Mag. Res., 1986, 70, 493.

5. a) Brunger, A. T.; Adams, P. D.; Clore, G. M.; Gros, P.; Kunstleve-Grosse, R. W.; Kuszewski, J.; Nilges, N.; Pannu, N. S.; Read, R. J.; Rice, L. M.; Simonson, T.; Warren, G. L. Acta Cryst., 1998, 54, 905. b) Brunger, A. T. Nature Protocols, 2007, 2, 2728.

6. All computational work was supported by the facilities and staff of Yale University Faculty of Arts and Sciences High Performance Computing Center, and by the National Science Foundation under grant \#CNS 08-21132 that partially funded acquisition of the facilities.

7. Gaussian 09, Revision D.01, Frisch, M. J.; Trucks, G. W.; Schlegel, H. B.; Scuseria, G. E.; Robb, M. A.; Cheeseman, J. R.; Scalmani, G.; Barone, V.; 
Mennucci, B.; Petersson, G. A.; Nakatsuji, H.; Caricato, M.; Li, X.; Hratchian, H. P.; Izmaylov, A. F.; Bloino, J.; Zheng, G.; Sonnenberg, J. L.; Hada, M.; Ehara, M.; Toyota, K.; Fukuda, R.; Hasegawa, J.; Ishida, M.; Nakajima, T.; Honda, Y.; Kitao, O.; Nakai, H.; Vreven, T.; Montgomery Jr., J. A.; Peralta, J. E.; Ogliaro, F.; Bearpark, M.; Heyd, J. J.; Brothers, E.; Kudin, K. N.; Staroverov, V. N.; Kobayashi, R.; Normand, J.; Raghavachari, K.; Rendell, A.; Burant, J. C.; lyengar, S. S.; Tomasi, J.; Cossi, M.; Rega, N.; Millam, J. M.; Klene, M.; Knox, J. E.; Cross, J. B.; Bakken, V.; Adamo, C.; Jaramillo, J.; Gomperts, R.; Stratmann, R. E.; Yazyev, O.; Austin, A. J.; Cammi, R.; Pomelli, C.; Ochterski, J. W.; Martin, R. L.; Morokuma, K.; Zakrzewski, V. G.; Voth, G. A.; Salvador, P.; Dannenberg, J. J.; Dapprich, S.; Daniels, A. D.; Farkas, Ö.; Foresman, J. B.; Ortiz, J. V.; Cioslowski, J.; Fox, D. J. Gaussian, Inc., Wallingford CT, 2009.

8. Tomasi, J.; Mennucci, B.; Cammi, R. Chem. Rev., 2005, 105, 2999. 